\title{
Copper-Mediated Synthesis of $N$-Acyl Vinylogous Carbamic Acids and Derivatives: Synthesis of the Antibiotic CJ-15,801
}

Chong Han, Ruichao Shen, Shun Su, and John A. Porco, Jr*

Department of Chemistry and

Center for Chemical Methodology and Library Development, Boston University, 590 Commonwealth Avenue,

Boston, Massachusetts 02215

Email:porco@chem.bu.edu

\section{Supporting Information}

General Information: ${ }^{1} \mathrm{H}$ NMR spectra were recorded on a $400 \mathrm{MHz}$ spectrometer at ambient temperature. ${ }^{13} \mathrm{C}$ NMR spectra were recorded on a $75.0 \mathrm{MHz}$ spectrometer at ambient temperature with complete proton decoupling. Chemical shifts are reported in parts per million relative to $\mathrm{CDCl}_{3}\left({ }^{1} \mathrm{H}, \delta 7.27 ;{ }^{13} \mathrm{C}, \delta 77.23\right)$ unless otherwise stated. Data for ${ }^{1} \mathrm{H} \mathrm{NMR}$ are reported as follows: chemical shift, integration, multiplicity (app = apparent, br = broad, ovrlp $=$ overlapping, $\mathrm{s}=$ singlet, $\mathrm{d}=$ doublet, $\mathrm{t}=$ triplet, $\mathrm{q}=$ quartet, $\mathrm{m}=$ multiplet) and coupling constants. Infrared spectra were recorded on a Nicolet Nexus 670 FT-IR spectrophotometer and a Nicolet Nexus 470 FT-IR + Continu $\mu \mathrm{M}$ microscope. Optical rotations were recorded on an AUTOPOL III digital polarimeter at $589 \mathrm{~nm}$, and are reported as $[\alpha]_{\mathrm{D}}$ (concentration in grams/100 mL solvent). HPLC analysis was performed on an Agilent 1100 series HPLC (column: Agilent, Eclipse XDB-C18, $5 \mu \mathrm{m}, 4.6 \times 150 \mathrm{~mm}$ ). HPLC-ELSD analysis was performed on an Agilent 1100 series LC/ELSD (Column: Waters, Xterra MS C $185 \mu \mathrm{m} 4.6 \times 100$ $\mathrm{mm}$ ). High-resolution mass spectra were obtained in the Boston University Mass Spectrometry Laboratory using a Finnegan MAT-90 spectrometer. HR-TOFMS spectra were obtained using a Micromass LCT spectrometer by Ms. Qing Liao (Department of Chemistry and Chemical Biology, Harvard University). Analytical thin layer chromatography was performed on 0.25 mm silica gel 60-F plates. Flash chromatography was performed using 200 400 mesh silica gel (Scientific Absorbent Incorporated) or neutral aluminum oxide (Sigma-Aldrich). Sep-Pak Vac 12 cc C-18 cartridges (Part NO. WAT036915) were obtained from Waters, Inc. Yields refer to chromatographically and spectroscopically pure materials, unless otherwise stated. Tetrahydrofuran was freshly distilled under argon from sodium/benzophenone ketyl. Copper (I) thiophene-2-carboxylate (CuTC) was prepared according the procedure of Allred and 
Liebeskind. ${ }^{1}$ Copper (I) tetrakis (acetonitrile) hexafluorophosphate $\left(\mathrm{Cu}\left(\mathrm{CH}_{3} \mathrm{CN}\right)_{4} \mathrm{PF}_{6}\right)$ was prepared according the procedure of Kubas. ${ }^{2}$ 2,6-Hexadienamide (sorbamide) was prepared according to the method reported by Pellegata. ${ }^{3}$ Dimethylacetamide (DMA) was distilled from barium oxide and stored over $4 \AA$ molecular sieves prior to use. PS- $\mathrm{NH}_{2}$, PS-HOBt, and chloro1,1,3,3-tetramethyluronium hexachloro-antimonate (ACTU) were obtained from Argonaut Technologies (Foster City, CA.). All other reagents were used as supplied by Sigma-Aldrich, Lancaster, Fluka, Strem, and Bachem Chemicals. Solid-phase synthesis reactions were conducted on the Quest 205 and 210 parallel synthesizers (Argonaut Technologies). All other reactions were performed in oven-dried glassware under an argon atmosphere unless otherwise noted.

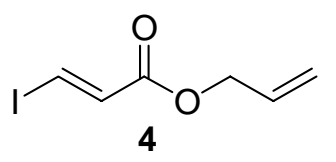

(E)-allyl-3-iodo acrylate 4: To a $250 \mathrm{~mL}$ flask were added $\beta$-iodo acrylic $\operatorname{acid}^{4}(11.88 \mathrm{~g}, 60.0 \mathrm{mmol})$, DMAP $(0.44 \mathrm{~g}, 3.6 \mathrm{mmol})$, and DMAP·HCl $(1.33 \mathrm{~g}, 8.4 \mathrm{mmol})$. Allyl alcohol $(20.4 \mathrm{~mL}, 300 \mathrm{mmol})$ and $\mathrm{CH}_{2} \mathrm{Cl}_{2}(40$ $\mathrm{mL}$ ) were added to the flask using oven-dried syringes. The solution was cooled to $0{ }^{\circ} \mathrm{C}$ and 1,3-diisopropylcarbodiimide (DIC) $(9.86 \mathrm{~mL}, 63.0 \mathrm{mmol})$ was added dropwise. The reaction was warmed to rt and stirred for $1 \mathrm{~h}$. The reaction mixture was filtered through Celite and concentrated. After dilution with EtOAc, the organic layer was washed with saturated $\mathrm{NaHCO}_{3}(2 \times)$, brine $(2 \times)$ and the aqueous layer extracted $2 \times$ with EtOAc. The organic layers were combined, dried over $\mathrm{Na}_{2} \mathrm{SO}_{4}$, and concentrated. Purification on silica gel (hexane: $\mathrm{EtOAc}=20: 1)$ provided $13.02 \mathrm{~g}(54.8 \mathrm{mmol}, 92 \%)$ of $(E)$-allyl- $\beta$-iodo acrylate 4 as a pale yellow oil. IR (neat): 3072, 2943, 1722, 1591, 1298, 1250, 1212, 1145, 991, $947 \mathrm{~cm}^{-1} ;{ }^{1} \mathrm{H}$ NMR: $\delta 7.92(1 \mathrm{H}, \mathrm{d}, J=15.2 \mathrm{~Hz}), 6.91(1 \mathrm{H}, \mathrm{d}, J=15.2 \mathrm{~Hz}), 5.96(1 \mathrm{H}, \mathrm{ddt}, J=17.2,10.4,5.6$ $\mathrm{Hz}), 5.35(1 \mathrm{H}, \mathrm{d}, J=17.2 \mathrm{~Hz}), 5.26(1 \mathrm{H}, \mathrm{d}, J=10.4 \mathrm{~Hz}), 4.64(2 \mathrm{H}, \mathrm{d}, J=5.6 \mathrm{~Hz}) \mathrm{ppm} ;{ }^{13} \mathrm{C}$ NMR: $\delta$ 164.0, 136.4, 131.9, 119.0, 100.1, 65.7 ppm; CI-HRMS calcd. for $\mathrm{C}_{6} \mathrm{H}_{7} \mathrm{IO}_{2}: 237.9491$, found 237.9520 .<smiles>O=C(/C=C/I)NCc1ccccc1</smiles>

(E)-allyl-3-iodo acrylamide 8: To a $100 \mathrm{~mL}$ flask were added $\beta$-iodo acrylic acid $^{4}(989.8 \mathrm{mg}, 5.0 \mathrm{mmol})$ and DMAP $(122.2 \mathrm{mg}, 1.0 \mathrm{mmol})$. Benzylamine $(819 \mu \mathrm{L}, 7.5 \mathrm{mmol})$ and $\mathrm{CH}_{2} \mathrm{Cl}_{2}(10 \mathrm{~mL})$ were added to the flask using oven-dried syringes. The solution was cooled to $0{ }^{\circ} \mathrm{C}$ and DIC ( $861 \mu \mathrm{L}, 5.5 \mathrm{mmol})$ was added dropwise. The reaction was warmed to rt, stirred for $6 \mathrm{~h}$, filtered through Celite, and concentrated. After dilution with ethyl acetate, the organic layer was washed with saturated $\mathrm{NaHCO}_{3}(2 \times)$, brine $(2 \times)$ and the aqueous layer extracted with EtOAc $(2 \times)$. The organic layers were combined, dried over $\mathrm{Na}_{2} \mathrm{SO}_{4}$, and concentrated. Purification on silica gel (hexane: EtOAc $=3: 1)$ provided $718 \mathrm{mg}(2.5 \mathrm{mmol}, 50 \%)$ of 3-iodo- $N$-benzyl-2propenamide 8 as pale yellow needles. mp. $165 \sim 167{ }^{\circ} \mathrm{C}$; IR (neat): 3291, 3055, 1631, 1592, 1546, 1452, 1309, 1234, 1151, 1024, 1009, $958 \mathrm{~cm}^{-1}$; ${ }^{1} \mathrm{H}$ NMR: $\delta 7.70(1 \mathrm{H}, \mathrm{br}$ s), $7.66(1 \mathrm{H}, \mathrm{d}, J$ $=14.4 \mathrm{~Hz}), 7.30 \sim 7.21(5 \mathrm{H}, \mathrm{m}), 7.11(1 \mathrm{H}, \mathrm{d}, J=14.4 \mathrm{~Hz}), 4.44(2 \mathrm{H}, \mathrm{d}, J=6.0 \mathrm{~Hz}) \mathrm{ppm} ;{ }^{13} \mathrm{C}$

\footnotetext{
${ }^{1}$ Allred, G. D.; Liebeskind, L. S. J. Am. Chem. Soc. 1996, 118, 2748.

${ }^{2}$ Kubas, G. J. Inorg. Synth. 1979, 19, 90.

${ }^{3}$ Pellegata, R.; Italia, A.; Villa, M. Synthesis, 1985, 517

${ }^{4}$ Takeuchi, R.; Tanabe, K.; Tanaka S. J. Org. Chem. 2000, 65, 1558
} 
NMR: $\delta 164.5,141.0,140.2,129.5$ (2C), 128.7 (2C), 128.1, 93.6, 43.6 ppm; CI-HRMS calcd. for $\mathrm{C}_{10} \mathrm{H}_{11} \mathrm{INO}[\mathrm{M}+\mathrm{H}]^{+}: 287.9885$, found 287.9894 .

\section{General Procedure for the Preparation of $N$-Acyl Vinylogous Carbamates and Ureas:}<smiles>C=CCOC(=O)/C=C/NC(=O)c1ccccc1</smiles>

Benzamide (90.9 mg, $0.75 \mathrm{mmol}$ ), $\mathrm{Rb}_{2} \mathrm{CO}_{3}$ (173.2 $\mathrm{mg}, 0.75$ $\mathrm{mmol}), \mathrm{Cu}\left(\mathrm{CH}_{3} \mathrm{CN}\right)_{4} \mathrm{PF}_{6}(9.3 \mathrm{mg}, 0.025 \mathrm{mmol})$, and $3,4,7,8-$ tetramethyl-1,10-phenanthroline $(11.8 \mathrm{mg}, 0.05 \mathrm{mmol})$ were placed in a flame-dried $10 \mathrm{~mL}$ Schlenk tube equipped with a stir bar. $\beta$-Iodo-acrylate $4(59.5 \mathrm{mg}, 0.25 \mathrm{mmol}, 36 \mu \mathrm{L})$ and anhydrous DMA $(1 \mathrm{~mL})$ were added to the Schlenk tube. The mixture was degassed under high vacuum until no further gas evolution was observed. The suspension was stirred at $45^{\circ} \mathrm{C}$ for $12 \mathrm{~h}$ under argon. The brown slurry was cooled to room temperature and diluted with $4 \mathrm{~mL}$ EtOAc and $1 \mathrm{~mL} \mathrm{pH} 7$ buffer. The EtOAc extract was then filtered through neutral alumina. The water layer was further extracted with a total of $20 \mathrm{~mL}$ EtOAc until the product was removed form the aqueous layer (monitored by TLC). The EtOAc extracts were washed with $\mathrm{pH} 7$ buffer $(1 \times)$ and brine $(1 \times)$ to remove DMA and the aqueous layer was further extracted with EtOAc $(2 \times)$ (this step is not necessary when using DME as solvent). The organic layers were combined, dried over anhydrous $\mathrm{Na}_{2} \mathrm{SO}_{4}$, and concentrated. Pure $N$-acyl vinylogous carbamate 5a was obtained as a white solid (32.0 mg, 54 \% yield; 41.2 mg, $71 \%$ yield using DME as solvent) after flash chromatography on silica gel (hexane: EtOAc = 3:1). mp. 111 113 ${ }^{\circ} \mathrm{C}$; IR (neat): 3314, 3084, 2935, 1678, 1634, 1518, 1488, 1448, 1361, 1302, 1212, 1143, 1030, $990 \mathrm{~cm}^{-1}$; ${ }^{1} \mathrm{H}$ NMR: $\delta 9.66(1 \mathrm{H}$, br d, $J=11.2 \mathrm{~Hz}), 8.27(1 \mathrm{H}, \mathrm{dd}$, $J=14.0,11.2 \mathrm{~Hz}), 7.84(2 \mathrm{H}, \mathrm{d}, J=7.6 \mathrm{~Hz}), 7.49(1 \mathrm{H}, \mathrm{dd}, J=7.6,7.6 \mathrm{~Hz}), 7.38(2 \mathrm{H}, \mathrm{dd}, J=$ 7.6, 7.6 Hz), $5.91(1 \mathrm{H}, \mathrm{ddt}, J=17.2,10.4,5.6 \mathrm{~Hz}), 5.72(1 \mathrm{H}, \mathrm{d}, J=14.0 \mathrm{~Hz}), 5.27(1 \mathrm{H}, \mathrm{d}, J=$ $17.2 \mathrm{~Hz}), 5.18(1 \mathrm{H}, \mathrm{d}, J=10.4 \mathrm{~Hz}), 4.57(2 \mathrm{H}, \mathrm{d}, J=5.6 \mathrm{~Hz}) \mathrm{ppm} ;{ }^{13} \mathrm{C}$ NMR: $\delta 167.8,166.0$, 139.2, 133.0, 132.4 (2C, ovrlp), 128.9 (2C), 127.9 (2C), 118.2, 102.2, 65.0 ppm; CI-HRMS calcd. for $\mathrm{C}_{13} \mathrm{H}_{14} \mathrm{NO}_{3}[\mathrm{M}+\mathrm{H}]^{+}:$232.0974, found 232.0993 .

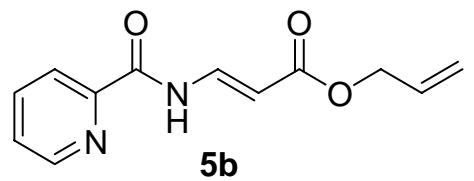

$N$-acyl vinylogous carbamate 5b: Prepared according to the general procedure as a pale yellow solid (43\% yield) after flash chromatography on silica gel (hexane: EtOAc = 3:1). mp. 114 116 ${ }^{\circ} \mathrm{C}$; IR (neat): 3279, 3084, 2938, 1695, 1631, 1503, 1358, 1315, 1294, 1272, 1213, 1142, $991 \mathrm{~cm}^{-1}$; ${ }^{1} \mathrm{H}$ NMR: $\delta 10.07(1 \mathrm{H}, \mathrm{br} \mathrm{d}, J$ $=12.4 \mathrm{~Hz}), 8.59(1 \mathrm{H}, \mathrm{d}, J=4.8 \mathrm{~Hz}), 8.22(1 \mathrm{H}, \mathrm{d}, J=8.0 \mathrm{~Hz}), 8.22(1 \mathrm{H}, \mathrm{dd}, J=14.0,12.4 \mathrm{~Hz})$, $7.91(1 \mathrm{H}, \mathrm{dd}, J=8.0,6,4 \mathrm{~Hz}), 7.51(1 \mathrm{H}, \mathrm{dd}, J=6.4,4.8 \mathrm{~Hz}), 5.99(1 \mathrm{H}, \mathrm{ddt}, J=17.2,10.4,5.6$ $\mathrm{Hz}), 5.76(1 \mathrm{H}, \mathrm{d}, J=14.0 \mathrm{~Hz}), 5.34(1 \mathrm{H}, \mathrm{d}, J=17.2 \mathrm{~Hz}), 5.23(1 \mathrm{H}, \mathrm{d}, J=10.4 \mathrm{~Hz}), 4.66(2 \mathrm{H}$, $\mathrm{d}, J=5.6 \mathrm{~Hz}) \mathrm{ppm} ;{ }^{13} \mathrm{C} \mathrm{NMR}: \delta 167.3,162.5,148.7,148.2,138.1,137.5,132.7,127.7,123.4$, 118.3, 103.1, 65.1 ppm; CI-HRMS calcd. for $\mathrm{C}_{12} \mathrm{H}_{13} \mathrm{~N}_{2} \mathrm{O}_{3}[\mathrm{M}+\mathrm{H}]^{+}:$233.0926, found 233.0901.

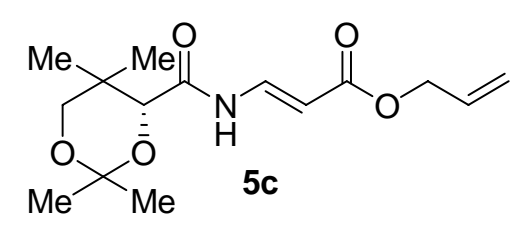

$\mathrm{N}$-acyl vinylogous carbamate 5c: Prepared according to the general procedure as a white crystalline solid ( $90 \%$ yield) after flash chromatography on neutral $\mathrm{Al}_{2} \mathrm{O}_{3}$ (hexane: EtOAc $=5: 1$ ). mp. 69 71 ${ }^{\circ} \mathrm{C}$; IR (neat): 3331, 3085, 2992, 2959, 2874, 1720, $1639,1495,1393,1378,1361,1301,1260,1200,1137,1098$, 1052, 1021, $994 \mathrm{~cm}^{-1}$; ${ }^{1} \mathrm{H}$ NMR: $\delta 8.43(1 \mathrm{H}, \mathrm{d}, J=11.6 \mathrm{~Hz})$, $8.00(1 \mathrm{H}, \mathrm{dd}, J=14.4,11.6 \mathrm{~Hz}), 5.95(1 \mathrm{H}, \mathrm{ddt}, J=17.2,10.4,5.6 \mathrm{~Hz}), 5.61(1 \mathrm{H}, \mathrm{d}, J=14.4$ $\mathrm{Hz}), 5.31(1 \mathrm{H}, \mathrm{d}, J=17.2 \mathrm{~Hz}), 5.20(1 \mathrm{H}, \mathrm{d}, J=10.4 \mathrm{~Hz}), 4.61(2 \mathrm{H}, \mathrm{d}, J=5.6 \mathrm{~Hz}), 4.16(1 \mathrm{H}, \mathrm{s})$, 
$3.69(1 \mathrm{H}, \mathrm{d}, J=12.0 \mathrm{~Hz}), 3.29(1 \mathrm{H}, \mathrm{d}, J=12.0 \mathrm{~Hz}), 1.47(3 \mathrm{H}, \mathrm{s}), 1.41(3 \mathrm{H}, \mathrm{s}), 1.01(3 \mathrm{H}, \mathrm{s})$, 0.97 (3H, s) ppm; ${ }^{13} \mathrm{C}$ NMR: $\delta 168.3,167.1,136.6,132.7,118.2,102.8,99.7,77.4,71.4,65.0$, 33.5, 29.5, 21.9, 18.9, 18.7 ppm; CI-HRMS calcd. for $\mathrm{C}_{15} \mathrm{H}_{24} \mathrm{NO}_{5}[\mathrm{M}+\mathrm{H}]^{+}: 298.1654$, found 298.1667. $[\alpha]_{\mathrm{D}}^{25}=+32.3\left(c=0.13, \mathrm{CHCl}_{3}\right)$

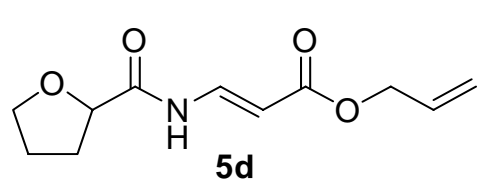

$N$-acyl vinylogous carbamate 5d: Prepared according to the general procedure as a colorless oil (61 \% yield) after flash chromatography on silica gel (hexane: EtOAc= 3:1). IR (neat): 3301, 2984, 2881, 1683, 1625, 1496, 1361, 1254, 1212, 1129, 1074, 1035, 991, 936, $851 \mathrm{~cm}^{-1}$; ${ }^{1} \mathrm{H}$ NMR: $\delta 8.67(1 \mathrm{H}, \mathrm{d}, J=$ $12.0 \mathrm{~Hz}), 7.98(1 \mathrm{H}, \mathrm{dd}, J=14.0,12.0 \mathrm{~Hz}), 5.96(1 \mathrm{H}, \mathrm{ddt}, J=17.2,10.4,5.6 \mathrm{~Hz}), 5.59(1 \mathrm{H}, \mathrm{d}, J$ $=14.0 \mathrm{~Hz}), 5.31(1 \mathrm{H}, \mathrm{d}, J=17.2 \mathrm{~Hz}), 5.21(1 \mathrm{H}, \mathrm{d}, J=10.4 \mathrm{~Hz}), 4.62(2 \mathrm{H}, \mathrm{d}, J=5.6 \mathrm{~Hz})$, 4.42(1H, dd, $J=8.4,6.0 \mathrm{~Hz}), 3.99 \sim 3.86(2 \mathrm{H}, \mathrm{m}), 2.35 \sim 2.26(1 \mathrm{H}, \mathrm{m}), 2.11 \sim 2.01(1 \mathrm{H}, \mathrm{m})$, 1.98 1.82 (2H, m) ppm; ${ }^{13} \mathrm{C}$ NMR: $\delta 172.1,167.1,136.9,132.7,118.3,102.9,78.3,69.9,65.1$, 30.2, 25.6 ppm; CI-HRMS calcd. for $\mathrm{C}_{11} \mathrm{H}_{16} \mathrm{NO}_{4}[\mathrm{M}+\mathrm{H}]^{+}: 226.1080$, found 226.1078.

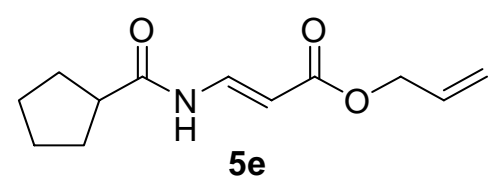

$N$-acyl vinylogous carbamate 5e: Prepared according to the general procedure as a colorless oil (6\% yield) after flash chromatography on silica gel (hexane: $\mathrm{EtOAc}=3: 1)$. IR (neat): 3292, 2959, 2871, 1717, 1690, 1633, 1514, 1452, 1377, 1306, $1247,1218,1145,988,928,845,724 \mathrm{~cm}^{-1} ;{ }^{1} \mathrm{H}$ NMR: $\delta 8.08$ $(1 \mathrm{H}, \mathrm{dd}, J=14.4,12.0 \mathrm{~Hz}), 7.71(1 \mathrm{H}$, br d, $J=12.0 \mathrm{~Hz}), 5.96(1 \mathrm{H}, \mathrm{ddt}, J=17.2,10.4,5.6 \mathrm{~Hz})$, $5.46(1 \mathrm{H}, \mathrm{d}, J=14.4 \mathrm{~Hz}), 5.32(1 \mathrm{H}, \mathrm{d}, J=17.2 \mathrm{~Hz}), 5.22(1 \mathrm{H}, \mathrm{d}, J=10.4 \mathrm{~Hz}), 4.62(2 \mathrm{H}, \mathrm{d}, J=$ $5.6 \mathrm{~Hz}), 2.63(1 \mathrm{H}, \mathrm{tt}, J=7.6,7.6 \mathrm{~Hz}), 1.90 \sim 1.57(8 \mathrm{H}, \mathrm{m}) \mathrm{ppm} ;{ }^{13} \mathrm{C} \mathrm{NMR}: \delta 175.0,167.6$, 138.6, 132.6, 118.3, 100.8, 65.0, 45.8, 30.3, 26.1 ppm; CI-HRMS calcd. for $\mathrm{C}_{12} \mathrm{H}_{18} \mathrm{NO}_{3}$ $[\mathrm{M}+\mathrm{H}]^{+}: 224.1287$, found 224.1285 .

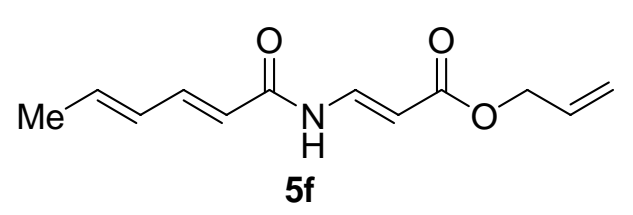

$\mathrm{N}$-acyl vinylogous carbamate 5f: Prepared according to the general procedure (DME as solvent) as a pale yellow solid (58\% yield) after flash chromatography on silica gel (hexane: $\mathrm{EtOAc}=3: 1$ ). $\mathrm{mp} .>250{ }^{\circ} \mathrm{C}$; IR (neat): 3273, 3080, 3012, 2953, 1714, 1683, 1635, 1559, 1540, 1507, 1457, 1342, 1295, 1256, 1130, $986 \mathrm{~cm}^{-1}$; ${ }^{1} \mathrm{H}$ NMR: $\delta 8.66(1 \mathrm{H}$, br d, $J=12.0 \mathrm{~Hz}), 8.17(1 \mathrm{H}, \mathrm{dd}$, $J=14.4,12.0 \mathrm{~Hz}), 7.35(1 \mathrm{H}, \mathrm{dd}, J=15.2,10.4 \mathrm{~Hz}), 6.18 \sim 6.16(2 \mathrm{H}, \mathrm{m}), 5.96(1 \mathrm{H}, \mathrm{ddt}, J=17.2$, 10.4, $5.6 \mathrm{~Hz}), 5.87(1 \mathrm{H}, \mathrm{d}, J=15.2 \mathrm{~Hz}), 5.49(1 \mathrm{H}, \mathrm{d}, J=14.4 \mathrm{~Hz}), 5.32(1 \mathrm{H}, \mathrm{d}, J=17.2 \mathrm{~Hz})$, $5.21(1 \mathrm{H}, \mathrm{d}, J=10.4 \mathrm{~Hz}), 4.62(2 \mathrm{H}, \mathrm{d}, J=5.6 \mathrm{~Hz}), 1.84(3 \mathrm{H}, \mathrm{d}, J=4.8 \mathrm{~Hz}) \mathrm{ppm} ;{ }^{13} \mathrm{C} \mathrm{NMR:} \delta$ $167.3,162.5,148.7,138.1,137.5,132.7,127.7,123.4,118.3,103.1,65.2,19.0$ ppm; CI-HRMS calcd. for $\mathrm{C}_{12} \mathrm{H}_{16} \mathrm{NO}_{3}[\mathrm{M}+\mathrm{H}]^{+}:$:222.1131, found 222.1145 .

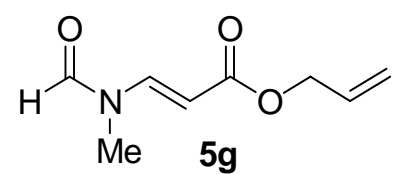

$\mathrm{N}$-acyl vinylogous carbamate 5g: Prepared according to the general procedure as a pale yellow oil (77 \% yield) after flash chromatography on silica gel (hexane: $\mathrm{EtOAc}=3: 1)$. IR (neat): 3400 , 3099, 2946, 1712, 1626, 1434, 1242, $1071 \mathrm{~cm}^{-1}$; ${ }^{1} \mathrm{H}$ NMR: (major rotamor) $\delta 8.45(1 \mathrm{H}, \mathrm{s}), 7.82(1 \mathrm{H}, \mathrm{d}, J=13.6 \mathrm{~Hz}), 5.98(1 \mathrm{H}, \mathrm{ddt}, J=17.2,10.4,5.6 \mathrm{~Hz}), 5.45$ $(1 \mathrm{H}, \mathrm{d}, J=13.6 \mathrm{~Hz}), 5.34(1 \mathrm{H}, \mathrm{d}, J=17.2 \mathrm{~Hz}), 5.25(1 \mathrm{H}, \mathrm{d}, J=10.4 \mathrm{~Hz}), 4.65(2 \mathrm{H}, \mathrm{d}, J=5.6$ 
$\mathrm{Hz}), 3.06(3 \mathrm{H}, \mathrm{s}) \mathrm{ppm} ;{ }^{13} \mathrm{C} \mathrm{NMR}:$ (major rotamor) $\delta 167.0,163.4,144.1,132.5,118.5,99.8$, 65.2, 27.8 ppm; CI-HRMS calcd. for $\mathrm{C}_{8} \mathrm{H}_{12} \mathrm{NO}_{3}[\mathrm{M}+\mathrm{H}]^{+}:$170.0817, found 170.0806.

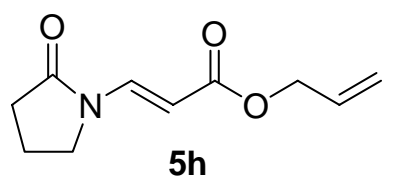

$N$-acyl vinylogous carbamate 5h: Prepared according to the general procedure as a pale yellow oil (92\% yield) after flash chromatography on silica gel (hexane: EtOAc= 3:1). IR (neat): 3084, 2944, 2894, 1731, 1629, 1483, 1461, 1401, 1359, 1327, 1217, 1153 $\mathrm{cm}^{-1} ;{ }^{1} \mathrm{H}$ NMR: $\delta 8.08(1 \mathrm{H}, \mathrm{d}, J=14.0 \mathrm{~Hz}), 5.94(1 \mathrm{H}, \mathrm{ddt}, J=17.2$, $10.4,5.6 \mathrm{~Hz}), 5.29(1 \mathrm{H}, \mathrm{d}, J=17.2 \mathrm{~Hz}), 5.19(1 \mathrm{H}, \mathrm{d}, J=14.0 \mathrm{~Hz}), 5.19(1 \mathrm{H}, \mathrm{d}, J=10.4 \mathrm{~Hz})$, $4.60(2 \mathrm{H}, \mathrm{d}, J=5.6 \mathrm{~Hz}), 3.53(2 \mathrm{H}, \mathrm{t}, J=7.2 \mathrm{~Hz}), 2.52(2 \mathrm{H}, \mathrm{t}, J=8.0 \mathrm{~Hz}), 2.17(2 \mathrm{H}, \mathrm{tt}, J=8.0$, $7.2 \mathrm{~Hz})$ ppm; ${ }^{13} \mathrm{C}$ NMR: $\delta 174.5,167.1,137.8,132.6,118.2,100.4,64.9,45.0,30.9,17.4$ ppm; CI-HRMS calcd. for $\mathrm{C}_{10} \mathrm{H}_{14} \mathrm{NO}_{3}[\mathrm{M}+\mathrm{H}]^{+}:$196.0974, found 196.0987.

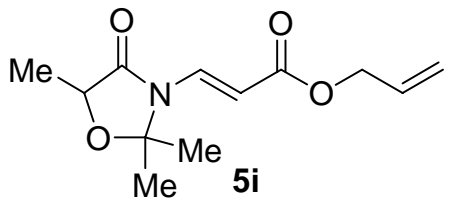

$N$-acyl vinylogous carbamate 5i: Prepared according to the general procedure as a white foam ( $72 \%$ yield) after flash chromatography on silica gel (hexane: EtOAc= $3: 1)$. IR (neat): $3375,2993,2942,1740,1635,1376,1222,992,940 \mathrm{~cm}^{-1} ;{ }^{1} \mathrm{H}$ NMR: $\delta 7.56(1 \mathrm{H}, \mathrm{d}, J=14.8 \mathrm{~Hz}), 6.00(1 \mathrm{H}, \mathrm{d}, J=14.8 \mathrm{~Hz}), 5.94$ $(1 \mathrm{H}, \mathrm{ddt}, J=17.2,10.4,5.6 \mathrm{~Hz}), 5.30(1 \mathrm{H}, \mathrm{d}, J=17.2 \mathrm{~Hz}), 5.20(1 \mathrm{H}, \mathrm{d}, J=10.4 \mathrm{~Hz}), 4.61(2 \mathrm{H}$, $\mathrm{d}, J=5.6 \mathrm{~Hz}), 4.38(1 \mathrm{H}, \mathrm{q}, J=6.8 \mathrm{~Hz}), 1.59(3 \mathrm{H}, \mathrm{s}), 1.50(3 \mathrm{H}, \mathrm{s}), 1.39(3 \mathrm{H}, \mathrm{d}, J=6.8 \mathrm{~Hz})$ ppm; ${ }^{13} \mathrm{C}$ NMR: $\delta 172.3,167.2,134.5,132.5,118.4,103.4,94.0,71.6,65.2,27.6,25.1,17.6$ ppm; CI-HRMS calcd. for $\mathrm{C}_{12} \mathrm{H}_{18} \mathrm{NO}_{4}[\mathrm{M}+\mathrm{H}]^{+}: 240.1236$, found 240.1253 .

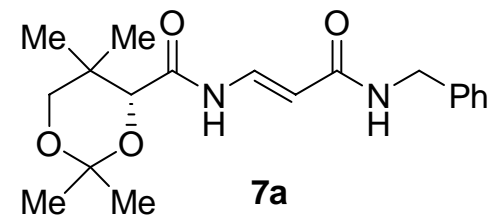

$N$-acyl vinylogous urea 7a: Prepared according to the general procedure as a white solid (70 \% yield) after flash chromatography on silica gel (hexane: $\mathrm{EtOAc}=1: 1)$. IR (neat): $3301,1657,1617,1498,1425,1327,1285,1257,1198,1175$, $1102,1045 \mathrm{~cm}^{-1} ;{ }^{1} \mathrm{H}$ NMR (acetone- $\left.d_{6}, 2.05\right): \delta 9.48(1 \mathrm{H}, \mathrm{br} \mathrm{d}, J$ $=10.8 \mathrm{~Hz}), 7.91(1 \mathrm{H}, \mathrm{dd}, J=14.0,10.8 \mathrm{~Hz}), 7.52(1 \mathrm{H}$, br t, $J=$ $5.6 \mathrm{~Hz}), 7.29 \sim 7.28(4 \mathrm{H}, \mathrm{m}), 7.23 \sim 7.20(1 \mathrm{H}, \mathrm{m}), 6.03(1 \mathrm{H}, \mathrm{d}, J=14.0 \mathrm{~Hz}), 4.44(2 \mathrm{H}, \mathrm{d}, J=5.6$ $\mathrm{Hz}), 4.27(1 \mathrm{H}, \mathrm{s}), 3.77(1 \mathrm{H}, \mathrm{d}, J=12.0 \mathrm{~Hz}), 3.28(1 \mathrm{H}, \mathrm{d}, J=12.0 \mathrm{~Hz}), 1.45(3 \mathrm{H}, \mathrm{s}), 1.38(3 \mathrm{H}$, s), $1.01(3 \mathrm{H}, \mathrm{s}), 0.99(3 \mathrm{H}, \mathrm{s}) \mathrm{ppm} ;{ }^{13} \mathrm{C} \mathrm{NMR}\left(\mathrm{CD}_{3} \mathrm{OD}, 49.15\right): \delta 169.6,167.2,141.0,134.2$, 129.4 (2C), 128.6 (2C), 127.9, 106.9, 100.1, 78.0, 71.7, 43.5, 33.9, 30.4, 22.1, 19.3, 19.0 ppm; CI-HRMS calcd. for $\mathrm{C}_{19} \mathrm{H}_{27} \mathrm{~N}_{2} \mathrm{O}_{4}[\mathrm{M}+\mathrm{H}]^{+}: 347.1971$, found 347.1985. $[\alpha]_{\mathrm{D}}{ }^{25}=+61.4(c=0.22$, $\mathrm{MeCN})$

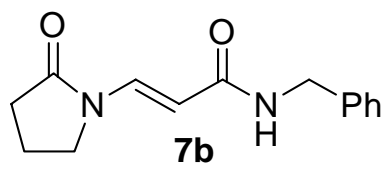

$N$-acyl vinylogous urea 7b: Prepared according to the general procedure as a white solid (72\% yield) after flash chromatography on silica gel (hexane: EtOAc: $\mathrm{MeOH}=20: 80: 5)$. mp. $173 \sim 174{ }^{\circ} \mathrm{C}$; IR (neat): 3276, 3096, 2961, 2932, 1717, 1665, 1653, 1602, 1558, 1407, 1330, $1249 \mathrm{~cm}^{-1} ;{ }^{1} \mathrm{H}$ NMR $\left(\mathrm{CD}_{3} \mathrm{OD}, 3.31\right): \delta 8.03(1 \mathrm{H}, \mathrm{d}, J=14.0$

$\mathrm{Hz}), 7.32 \sim 7.24(5 \mathrm{H}, \mathrm{m}), 5.91(1 \mathrm{H}$, br t, $J=5.6 \mathrm{~Hz}), 5.28(1 \mathrm{H}, \mathrm{d}, J=14.0 \mathrm{~Hz}), 4.49(2 \mathrm{H}, \mathrm{d}, J=$ $5.6 \mathrm{~Hz}), 3.51(2 \mathrm{H}, \mathrm{t}, J=7.2 \mathrm{~Hz}), 2.51(2 \mathrm{H}, \mathrm{t}, J=8.4 \mathrm{~Hz}), 2.14(2 \mathrm{H}, \mathrm{tt}, J=8.4,7.2 \mathrm{~Hz}) \mathrm{ppm}$; ${ }^{13} \mathrm{C}$ NMR (acetone- $\left.d_{6}, 29.92\right): \delta 175.0,166.9,141.1,134.8,129.4(2 \mathrm{C}), 128.6$ (2C), 127.9, 104.2, 45.8, 43.6, 31.4, 18.2 ppm; CI-HRMS calcd. for $\mathrm{C}_{14} \mathrm{H}_{16} \mathrm{~N}_{2} \mathrm{O}_{2}$ [M] $]^{+}: 244.1212$, found 244.1212 . 


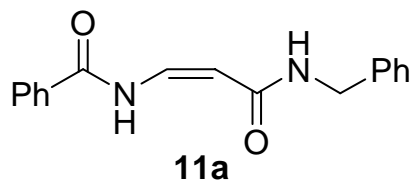

$N$-acyl vinylogous urea 11a: Prepared according to the general procedure as a yellow solid (25\% yield) after flash chromatography on silica gel $\left(\mathrm{CH}_{2} \mathrm{Cl}_{2}: \mathrm{MeOH}=20: 1\right)$. mp. $151 \sim 154{ }^{\circ} \mathrm{C}$; IR (neat): 3295, 2925, 1649, 1613, 1454, 1389, $1225 \mathrm{~cm}^{-1}$; ${ }^{1} \mathrm{H}$ NMR $\left(\mathrm{CD}_{3} \mathrm{OD}\right.$, 3.31): $\delta 7.95(2 \mathrm{H}, \mathrm{d}, J=6.8 \mathrm{~Hz}), 7.64(1 \mathrm{H}, \mathrm{dd}, J=6.8,6.8 \mathrm{~Hz})$, $7.60(2 \mathrm{H}, \mathrm{dd}, J=6.8,6.8 \mathrm{~Hz}), 7.55(1 \mathrm{H}, \mathrm{d}, J=8.8 \mathrm{~Hz}), 7.33 \sim 7.24(5 \mathrm{H}, \mathrm{m}), 5.44(1 \mathrm{H}, \mathrm{d}, J=8.8$ $\mathrm{Hz}), \quad 4.50(2 \mathrm{H}, \mathrm{s}) \mathrm{ppm} ;{ }^{13} \mathrm{C} \mathrm{NMR}$ (acetone- $\left.d_{6}, 29.92\right): \delta 170.1,164.7,140.4,136.3,134.0$, 133.7, 130.1 (2C), 129.5 (2C), 128.7 (2C), 128.5 (2C), 128.1, 101.2, 43.3 ppm; CI-HRMS calcd. for $\mathrm{C}_{17} \mathrm{H}_{16} \mathrm{~N}_{2} \mathrm{O}_{2}[\mathrm{M}]^{+}: 280.1212$, found 280.1210 .

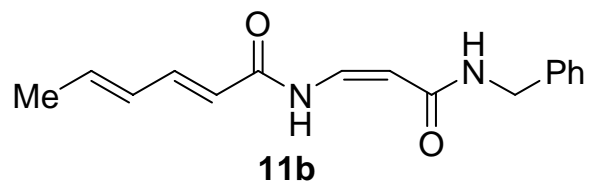

$N$-acyl vinylogous urea 11b: Prepared according to the general procedure as a yellow solid (18\% yield) after flash chromatography on silica gel $\left(\mathrm{CH}_{2} \mathrm{Cl}_{2}: \mathrm{CH}_{3} \mathrm{OH}=20: 1\right)$. mp. ${ }^{\circ}$; ; IR (neat): 3300, 3088, 3030, 2964, 2931, 1657, $1612,1556,1464,1394,1358,1238,1147,1100,1081$, $999 \mathrm{~cm}^{-1} ;{ }^{1} \mathrm{H}$ NMR (acetone- $\left.d_{6}, 2.05\right): \delta 11.46(1 \mathrm{H}, \mathrm{d}, J=10.4 \mathrm{~Hz}), 7.77(1 \mathrm{H}, \mathrm{br} \mathrm{s}), 7.39(1 \mathrm{H}$, $\mathrm{dd}, J=10.4,8.8 \mathrm{~Hz}), 7.31 \sim 7.27(5 \mathrm{H}, \mathrm{m}), 7.27(1 \mathrm{H}, \mathrm{dd}, J=14.8,10.4 \mathrm{~Hz}), 6.36(1 \mathrm{H}, \mathrm{dd}, J=$ $14.8,10.4 \mathrm{~Hz}), 6.27(1 \mathrm{H}, \mathrm{dq}, J=14.8,6.4 \mathrm{~Hz}), 6.10(1 \mathrm{H}, \mathrm{d}, J=14.8 \mathrm{~Hz}), 5.28(1 \mathrm{H}, \mathrm{d}, J=8.8$ $\mathrm{Hz}), 4.45(1 \mathrm{H}, \mathrm{d}, J=6.0 \mathrm{~Hz}), 1.85(1 \mathrm{H}, \mathrm{d}, J=6.4 \mathrm{~Hz}) \mathrm{ppm} ;{ }^{13} \mathrm{C}$ NMR (acetone- $\left.d_{6}, 29.92\right): \delta$ $169.7,164.4,144.3,140.6,140.2,135.9,131.1,129.5,128.6,128.1,122.5,100.2,43.2,18.8$ ppm; CI-HRMS calcd. for $\mathrm{C}_{16} \mathrm{H}_{18} \mathrm{~N}_{2} \mathrm{O}_{2}[\mathrm{M}]^{+}: 270.1368$, found 270.1381 .

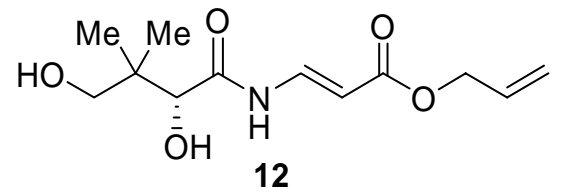

$N$-acyl vinylogous carbamate 12: To a test tube were added acetonide 5c $(80.1 \mathrm{mg}, 0.269 \mathrm{mmol})$ and $\mathrm{BiCl}_{3}(17.0 \mathrm{mg}$, $0.0539 \mathrm{mmol})$, followed by distilled $\mathrm{H}_{2} \mathrm{O}(96.9 \mathrm{mg}, 5.38$ mmol, $96.9 \mathrm{uL})$ and $\mathrm{MeCN}(1.1 \mathrm{~mL})$. The reaction was stirred at room temperature for $8 \mathrm{~h}$, then filtered and concentrated. After dilution with EtOAc, the reaction mixture was washed with sat. $\mathrm{NaHCO}_{3}$ $(2 \times)$ and the aqueous layer was extracted with EtOAc $(2 \times)$. The organic layers were combined, dried over $\mathrm{Na}_{2} \mathrm{SO}_{4}$, and concentrated. Purification using a Sep-Pak C18 cartridge (20\% $\left.\mathrm{MeCN} / \mathrm{H}_{2} \mathrm{O}\right)$ provided $52.0 \mathrm{mg}(75 \%)$ of 12 as a pale yellow solid. mp. $62 \sim 64{ }^{\circ} \mathrm{C}$; IR (neat): 3295, 2969, 2879, 1697, 1634, 1504, 1366, 1305, 1262, 1212, 1146, 1079, 1037, $989 \mathrm{~cm}^{-1} ;{ }^{1} \mathrm{H}$ NMR (CD $\left.{ }_{3} \mathrm{OD}, 3.31\right): \delta 7.99(1 \mathrm{H}, \mathrm{d}, J=14.4 \mathrm{~Hz}), 6.01(1 \mathrm{H}, \mathrm{ddt}, J=17.2,10.4,5.6 \mathrm{~Hz}), 5.78$ $(1 \mathrm{H}, \mathrm{d}, J=14.4 \mathrm{~Hz}), 5.34(1 \mathrm{H}, \mathrm{d}, J=17.2 \mathrm{~Hz}), 5.22(1 \mathrm{H}, \mathrm{d}, J=10.4 \mathrm{~Hz}), 4.63(2 \mathrm{H}, \mathrm{d}, J=5.6$ $\mathrm{Hz}), 4.02(1 \mathrm{H}, \mathrm{s}), 3.50(1 \mathrm{H}, \mathrm{d}, J=10.8 \mathrm{~Hz}), 3.39(1 \mathrm{H}, \mathrm{d}, J=10.8 \mathrm{~Hz}), 0.93(3 \mathrm{H}, \mathrm{s}), 0.92(3 \mathrm{H}$, s) ppm; ${ }^{13} \mathrm{C} \mathrm{NMR}\left(\mathrm{CD}_{3} \mathrm{OD}, 49.15\right): \delta 175.3,169.6,139.3,134.2,118.4,102.7,77.2,69.9,66.0$, 40.9, 21.6, 20.6 ppm; CI-HRMS calcd. for $\mathrm{C}_{12} \mathrm{H}_{20} \mathrm{NO}_{5}[\mathrm{M}+\mathrm{H}]^{+}$: 258.1341, found 258.1322. $[\alpha]_{\mathrm{D}}^{25}=+80.0(c=0.12, \mathrm{MeCN})$

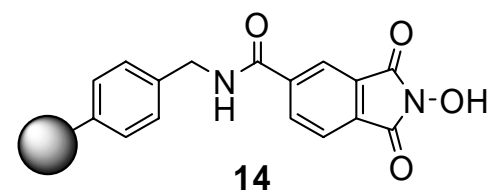

$\mathrm{N}$-hydroxylpthalimide resin 14: Pre-swelled $\mathrm{PS}-\mathrm{NH}_{2}$ resin $(5.0$ g, $6.50 \mathrm{mmol}$, loading: $1.30 \mathrm{mmol} / \mathrm{g}$,) was mixed with $40 \mathrm{~mL}$ $\mathrm{CH}_{2} \mathrm{Cl}_{2}$ in an $80 \mathrm{~mL}$ reaction vessel on the Quest 205 parallel synthesizer. After addition of $8.1 \mathrm{~mL} \mathrm{HCl}$ in dioxane $(4.0 \mathrm{M}$, $32.4 \mathrm{mmol})$, the mixture was agitated for $1 \mathrm{~h}$. Upon filtration, 
the PS- $\mathrm{NH}_{2} \cdot \mathrm{HCl}$ resin was washed with $\mathrm{CH}_{2} \mathrm{Cl}_{2}(3 \times 30 \mathrm{~mL})$ and transferred to a pre-silylated $250 \mathrm{~mL}$ two-neck flask equipped with a mechanical stirrer. Trimellitic anhydride chloride (6.80 $\mathrm{g}, 32.4 \mathrm{mmol}$ ) was added followed by $30 \mathrm{~mL} \mathrm{CH}_{2} \mathrm{Cl}_{2}$ and the reaction was cooled to $0^{\circ} \mathrm{C}$ using an ice bath. A solution of $\mathrm{Et}_{3} \mathrm{~N}(6.22 \mathrm{~mL}, 45.5 \mathrm{mmol})$ in $20 \mathrm{~mL} \mathrm{CH}_{2} \mathrm{Cl}_{2}$ was added to the reaction mixture over $2 \mathrm{~h}$ via syringe pump. The reaction was stirred at $0{ }^{\circ} \mathrm{C}$ for an additional 3 $\mathrm{h}$, warmed to $\mathrm{rt}$, and stirred for an additional $2 \mathrm{~h}$. After the reaction mixture was transferred to a reaction vessel on the Quest 205 synthesizer and the resulting anhydride resin was washed with $\mathrm{CH}_{2} \mathrm{Cl}_{2}(2 \times 20 \mathrm{~mL})$, DMF $(2 \times 20 \mathrm{~mL})$, THF $(2 \times 20 \mathrm{~mL})$, and $\mathrm{CH}_{2} \mathrm{Cl}_{2}(2 \times 20 \mathrm{~mL})$. Following washing, $\mathrm{NH}_{2} \mathrm{OH} \cdot \mathrm{HCl}(2.24 \mathrm{~g}, 32.5 \mathrm{mmol}, 5.0$ equiv.) and $40 \mathrm{~mL} \mathrm{3:1}$ pyridine: 1,2dichloroethane were added and the reaction was agitated for $24 \mathrm{~h}$ at $75^{\circ} \mathrm{C}$. The reaction was cooled to rt, the resin 14 filtered, washed with $\mathrm{MeOH}(2 \times 20 \mathrm{~mL}), \mathrm{DMF}(2 \times 20 \mathrm{~mL}), \mathrm{DMF}$ : $\mathrm{H}_{2} \mathrm{O}=1: 1(2 \times 30 \mathrm{~mL}), \mathrm{DMF}(1 \times 20 \mathrm{~mL})$, THF $(3 \times 20 \mathrm{~mL}), \mathrm{CH}_{2} \mathrm{Cl}_{2}(2 \times 20 \mathrm{~mL}), \mathrm{Et}_{2} \mathrm{O}(2 \times 20 \mathrm{~mL})$, and dried under high vacuum at $50{ }^{\circ} \mathrm{C}$ for $12 \mathrm{~h}$. IR (single bead): $3385,3060,3027,2925,1788$, 1728, 1669, 1601, 1514, 1494, 1453, 1374, 1299, 1194, 1141, 1115, 1021, 998, 913, 827, 761, $709 \mathrm{~cm}^{-1}$

Loading determination: Resin $14\left(100 \mathrm{mg}\right.$ ) was pre-swelled with $\mathrm{CH}_{2} \mathrm{Cl}_{2}$ in a $5 \mathrm{~mL}$ reaction vessel on the Quest-210 synthesizer and 4-biphenylmethanol (55.2 $\mathrm{mg}, 0.30 \mathrm{mmol})$ and azodicarboxylic acid bis[dimethylamide] ${ }^{5}(51.6 \mathrm{mg}, 0.30 \mathrm{mmol})$ were added as solids under $\mathrm{N}_{2}$ followed by $2 \mathrm{~mL} \mathrm{1:1} \mathrm{THF:} \mathrm{CH}_{2} \mathrm{Cl}_{2}$. After mixing to dissolve the reagents, $\mathrm{P}(n-\mathrm{Bu})_{3}(43.2 \mu \mathrm{L}$, $0.30 \mathrm{mmol}$ ) was added and reaction mixture was agitated for $6 \mathrm{~h}$. After filtering the reaction mixture, the resulting resin was washed with $\mathrm{DMF}(2 \times 3 \mathrm{~mL}), \mathrm{MeOH}(2 \times 3 \mathrm{~mL}), \mathrm{THF}(33 \times 3$ $\mathrm{mL}), \mathrm{CH}_{2} \mathrm{Cl}_{2}(3 \times 3 \mathrm{~mL})$. Following the addition of $2 \mathrm{~mL} \mathrm{CH} \mathrm{Cl}_{2}, \mathrm{NH}_{2} \mathrm{NH}_{2}(6.3 \mu \mathrm{L}, 0.20 \mathrm{mmol})$ was added and the reaction was agitated for $1 \mathrm{~h}$. The filtration was collected and treated with 2 $\mathrm{mL}$ acetone. After concentration, the resulting sample was subjected to an HPLC analysis using an Agilent 1100 with diodearray detector (DAD) and an Agilent Zorbax C18 $(3.5 \mu \mathrm{m}, 4.6 \times 75$ $\mathrm{mm}$ ) column (4-Biphenylmethanol as internal standard). The loading was determined to be $75 \%$ (0.77 $\mathrm{mmol} / \mathrm{g})$.

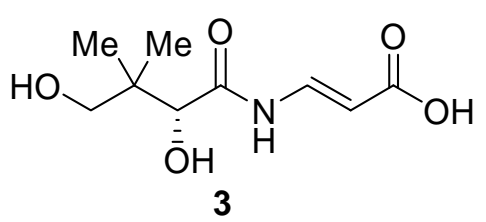

CJ-15,801 3: To a Teflon vial were added allyl ester 12 (13.2 $\mathrm{mg}, 0.0513 \mathrm{mmol})$, polymer supported $N$-hydroxypthalimide $\mathbf{1 4}$ (200 mg, $0.154 \mathrm{mmol}$, loading: $0.77 \mathrm{mmol} / \mathrm{g})$, and THF (1.5 $\mathrm{mL})$. After stirring for 5 minutes, $\mathrm{Pd}\left(\mathrm{PPh}_{3}\right)_{4}(11.9 \mathrm{mg}, 0.0103$ mmol) was added. The reaction was stirred in the dark at $35^{\circ} \mathrm{C}$ for $12 \mathrm{~h}$, then filtered and concentrated to provide crude product 3. Purification using a Sep-Pak C18 cartridge $\left(10 \% \mathrm{MeCN} /\right.$ deionized $\left.\mathrm{H}_{2} \mathrm{O}\right)$ afforded $8.9 \mathrm{mg}(80 \%)$ of acid 3 as a white amorphous solid. IR (neat): 3307, 2968, 2936, 2880, 1776, 1685, 1639, 1508, 1477, 1377, $1265,1210,1166,1080,1042,988,915,859 \mathrm{~cm}^{-1} ;{ }^{1} \mathrm{H}$ NMR $\left(\mathrm{CD}_{3} \mathrm{OD}, 3.31\right): \delta 7.96(1 \mathrm{H}, \mathrm{d}, J=$ $14.4 \mathrm{~Hz}), 5.70(1 \mathrm{H}, \mathrm{d}, J=14.4 \mathrm{~Hz}), 4.02(1 \mathrm{H}, \mathrm{s}), 3.50(1 \mathrm{H}, \mathrm{d}, J=10.8 \mathrm{~Hz}), 3.39(1 \mathrm{H}, \mathrm{d}, J=$ $10.8 \mathrm{~Hz}), 0.93(3 \mathrm{H}, \mathrm{s}), 0.92(3 \mathrm{H}, \mathrm{s}) \mathrm{ppm} ;{ }^{13} \mathrm{C} \mathrm{NMR}\left(\mathrm{CD}_{3} \mathrm{OD}, 49.15\right): \delta 175.3,172.0,139.3$, 103.5, 77.3, 70.0, 40.9, 21.6, 20.5 ppm; FAB-HRMS calcd. for $\mathrm{C}_{9} \mathrm{H}_{16} \mathrm{NO}_{5}[\mathrm{M}+\mathrm{H}]^{+}: 218.1028$, found 218.1029; $[\alpha]_{\mathrm{D}}{ }^{25}=+54.3(c=0.44, \mathrm{MeOH})$.

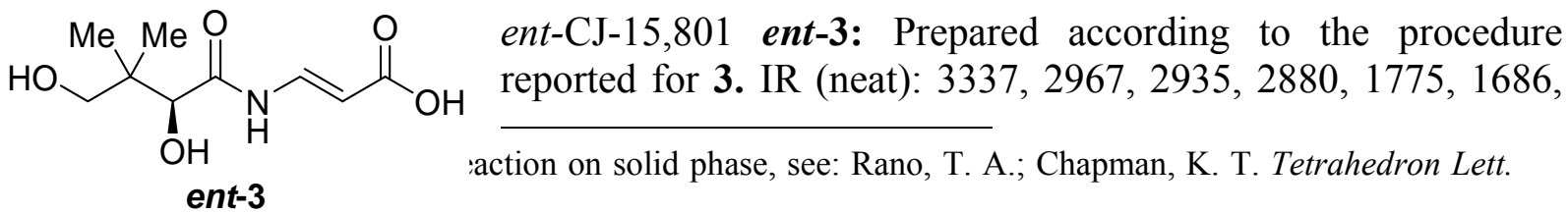


$1640,1510,1478,1380,1265,1211,1165,1079,1042,987,859 \mathrm{~cm}^{-1} ;{ }^{1} \mathrm{H}$ NMR $\left(\mathrm{CD}_{3} \mathrm{OD}\right.$, 3.31): $\delta 7.96(1 \mathrm{H}, \mathrm{d}, J=14.4 \mathrm{~Hz}), 5.70(1 \mathrm{H}, \mathrm{d}, J=14.4 \mathrm{~Hz}), 4.02(1 \mathrm{H}, \mathrm{s}), 3.50(1 \mathrm{H}, \mathrm{d}, J=10.8$ $\mathrm{Hz}), 3.39(1 \mathrm{H}, \mathrm{d}, J=10.8 \mathrm{~Hz}), 0.93(3 \mathrm{H}, \mathrm{s}), 0.92(3 \mathrm{H}, \mathrm{s}) \mathrm{ppm} ;{ }^{13} \mathrm{C}$ NMR $\left(\mathrm{CD}_{3} \mathrm{OD}, 49.15\right): \delta$ 175.3, 172.0, 139.3, 103.5, 77.3, 70.0, 40.9, 21.6, 20.5 ppm; CI-HRMS calcd. for $\mathrm{C}_{9} \mathrm{H}_{15} \mathrm{NO}_{5}$ $[\mathrm{M}]^{+}: 217.0950$, found 217.0960; $[\alpha]_{\mathrm{D}}^{25}=-49.3(c=0.28, \mathrm{MeOH})$.<smiles>O=C(O)/C=C/NC(=O)c1ccccc1</smiles>

$N$-acyl vinylogous carbamic acid 6a: Prepared from 5a in $78 \%$ yield according to the procedure reported for 3. IR (neat): 3332, 1670, 1640, $1554,1524,1411,1198,1142,988,965,843,804,723 \mathrm{~cm}^{-1} ;{ }^{1} \mathrm{H}$ NMR $\left(\mathrm{CD}_{3} \mathrm{OD}, 3.31\right): \delta 8.21(1 \mathrm{H}, \mathrm{d}, J=14.0 \mathrm{~Hz}), 7.95(2 \mathrm{H}, \mathrm{d}, J=7.2 \mathrm{~Hz})$, $7.61(1 \mathrm{H}, \mathrm{dd}, J=7.2,7.2 \mathrm{~Hz}), 7.54(2 \mathrm{H}, \mathrm{dd}, J=7.2,7.2 \mathrm{~Hz}), 5.76(1 \mathrm{H}, \mathrm{d}, J=14.0 \mathrm{~Hz}) \mathrm{ppm}$; ${ }^{13} \mathrm{C}$ NMR $\left(\mathrm{CD}_{3} \mathrm{OD}, 49.15\right)$ : $\delta 172.06,168.2$, 140.5, 134.3, 134.2, 130.1, 129.3, 103.9 ppm; CIHRMS calcd. for $\mathrm{C}_{10} \mathrm{H}_{10} \mathrm{NO}_{3}[\mathrm{M}+\mathrm{H}]^{+}:$192.0661, found 192.0640.

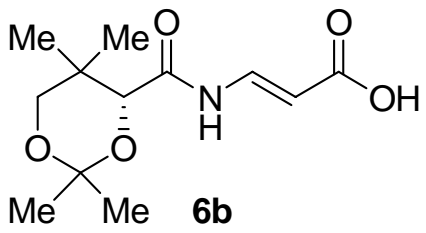

$N$-acyl vinylogous carbamic acid 6b: Prepared from 5c in $82 \%$ yield according to the procedure reported for 3. IR (neat): 3397 , 2992, 2958, 2875, 1693, 1639, 1504, 1440, 1381, 1262, 1200, 1161, 1097, 1051, 1021, 990, 948, $870 \mathrm{~cm}^{-1} ;{ }^{1} \mathrm{H}$ NMR $\left(\mathrm{CD}_{3} \mathrm{OD}\right.$, 3.31): $\delta 7.89(1 \mathrm{H}, \mathrm{d}, J=14.0 \mathrm{~Hz}), 5.83(1 \mathrm{H}, \mathrm{d}, J=14.0 \mathrm{~Hz}), 4.25$ $(1 \mathrm{H}, \mathrm{s}), 3.76(1 \mathrm{H}, \mathrm{d}, J=11.2 \mathrm{~Hz}), 3.28(1 \mathrm{H}, \mathrm{d}, J=11.2 \mathrm{~Hz}), 1.48$ $(3 \mathrm{H}, \mathrm{s}), 1.44(3 \mathrm{H}, \mathrm{s}), 0.99(3 \mathrm{H}, \mathrm{s}), 0.98(3 \mathrm{H}, \mathrm{s}) \mathrm{ppm} ;{ }^{13} \mathrm{C} \mathrm{NMR}\left(\mathrm{CD}_{3} \mathrm{OD}, 49.15\right): \delta 172.7,171.2$, 138.1, 105.5, 101.0, 78.8, 72.4, 34.4, 29.6, 22.3, 19.4, 19.1 ppm; CI-HRMS calcd. for $\mathrm{C}_{12} \mathrm{H}_{19} \mathrm{NO}_{5}[\mathrm{M}+\mathrm{H}]^{+}: 257.1263$, found $257.1251 ;[\alpha]_{\mathrm{D}}{ }^{25}=+63.4(c=0.41, \mathrm{MeCN})$

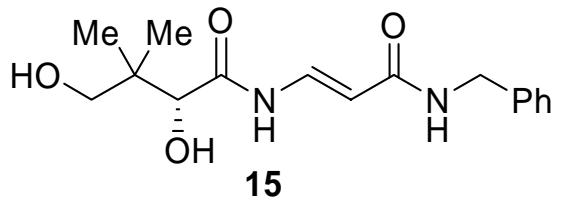

$N$-acyl vinylogous urea 15: Prepared from 7a according to the procedure reported for 12. Purification using a Sep-Pak C18 cartridge $\left(20-40 \% \mathrm{MeCN} / \mathrm{H}_{2} \mathrm{O}\right)$ provided $15(75 \%)$ as a pale yellow foam. IR (neat): 3300, 2965, 2931, 1710, $1660,1605,1496,1328,1255,1211,1082,1042 \mathrm{~cm}^{-1} ;{ }^{1} \mathrm{H}$ NMR (acetone- $\left.d_{6}, 2.05\right): \delta 9.65(1 \mathrm{H}, \mathrm{d}, J=10.8 \mathrm{~Hz}), 7.94$ $(1 \mathrm{H}, \mathrm{dd}, J=14.0,10.8 \mathrm{~Hz}), 7.63(1 \mathrm{H}, \mathrm{br} \mathrm{s}), 7.29(4 \mathrm{H}, \mathrm{m}), 7.22(1 \mathrm{H}, \mathrm{m}), 6.08(1 \mathrm{H}, \mathrm{d}, J=14.0$ $\mathrm{Hz}), 4.44(2 \mathrm{H}, \mathrm{d}, J=6.0 \mathrm{~Hz}), 4.08(1 \mathrm{H}, \mathrm{s}), 3.49(1 \mathrm{H}, \mathrm{d}, J=10.8 \mathrm{~Hz}), 3.40(1 \mathrm{H}, \mathrm{d}, J=10.8 \mathrm{~Hz})$, $0.92(3 \mathrm{H}, \mathrm{s}), 0.91(3 \mathrm{H}, \mathrm{s}) \mathrm{ppm} ;{ }^{13} \mathrm{C}$ NMR (acetone- $\left.d_{6}, 29.92\right): \delta 173.4,167.6,141.0,134.6$, 129.4 (2C), 128.6 (2C), 127.9, 106.3, 77.4, 70.3, 43.5, 40.3, 21.4, 20.7 ppm; CI-HRMS calcd. for $\mathrm{C}_{16} \mathrm{H}_{23} \mathrm{~N}_{2} \mathrm{O}_{4}[\mathrm{M}+\mathrm{H}]^{+}: 307.1658$, found 307.1639; $[\alpha]_{\mathrm{D}}{ }^{25}=+43.8(c=0.24, \mathrm{MeOH})$<smiles>O=C(/C=C/NC(=O)c1ccccc1)NCc1ccccc1</smiles>

$\mathrm{N}$-acyl vinylogous urea 7c: To a Teflon vial were added carbamic acid 6a (17.3 mg, $0.09 \mathrm{mmol}$ ), PS-HOBt resin (53.6 mg, $0.6 \mathrm{mmol}$, loading: $\quad 1.12 \mathrm{mmol} / \mathrm{g})$, chloro-1,1,3,3-tetramethyluronium hexachloro-antimonate (ACTU) (28.2 $\mathrm{mg}, 0.06 \mathrm{mmol})$, and DMF $(0.9 \mathrm{~mL})$. After stirring for 5 minutes at $0^{\circ} \mathrm{C}, 2,6$-lutidine $(32.1 \mathrm{mg}$, $0.30 \mathrm{mmol}, 35 \mu \mathrm{L}$ ) was added. The mixture was stirred at $\mathrm{rt}$ for $4 \mathrm{~h}$. The product resin 16 was then washed with DMF $(2 \times 2 \mathrm{~mL})$, THF $(2 \times 2 \mathrm{~mL}), \mathrm{CH}_{2} \mathrm{Cl}_{2}(2 \times 2 \mathrm{~mL})$, and then used immediately in the next step. A solution of benzylamine $(3.2 \mathrm{mg}, 0.03 \mathrm{mmol}, 3.3$ $\mu \mathrm{L}$ in $0.6 \mathrm{~mL}$ THF) was added to the activated resin 16 and the reaction stirred for $2 \mathrm{~h}$. The 
mixture was filtered and the resin was washed with DMF. Removal of solvent using high vacuum afforded $6.8 \mathrm{mg}(81 \%)$ of urea $7 \mathbf{c}$ as a white solid (Purity: $93 \%$ by HPLC-ELSD analysis). IR (neat): $3301,1649,1605,1514,1328,1291,1184 \mathrm{~cm}^{-1} ;{ }^{1} \mathrm{H}$ NMR $\left(\mathrm{CD}_{3} \mathrm{OD}, 3.31\right)$ : $\delta 8.12(1 \mathrm{H}, \mathrm{d}, J=14.0 \mathrm{~Hz}), 7.91(2 \mathrm{H}, \mathrm{d}, J=8.0 \mathrm{~Hz}), 7.60(1 \mathrm{H}, \mathrm{dd}, J=8.0,8.0 \mathrm{~Hz}), 7.51(2 \mathrm{H}$, $\mathrm{dd}, J=8.0,8.0 \mathrm{~Hz}), 7.30 \sim 7.28(4 \mathrm{H}, \mathrm{m}), 7.23(1 \mathrm{H}, \mathrm{dd}, J=7.2,7.2 \mathrm{~Hz}), 6.02(1 \mathrm{H}, \mathrm{d}, J=14.0$ $\mathrm{Hz}), 4.43(2 \mathrm{H}, \mathrm{s}) \mathrm{ppm} ;{ }^{13} \mathrm{C}$ NMR (DMSO- $\left.d_{6}, 39.51\right): \delta 166.1,165.3,140.0,134.7,133.0$, 132.6, 128.8 (2C), 128.5 (2C), 128.1 (2C), 127.5 (2C), 126.9, 105.7, 42.1; CI-HRMS calcd. for $\mathrm{C}_{17} \mathrm{H}_{16} \mathrm{~N}_{2} \mathrm{O}_{2}[\mathrm{M}+\mathrm{H}]^{+}: 280.1212$, found 280.1205 . 


\section{NMR data (in $\mathrm{CD}_{3} \mathrm{OD}$ ) comparison of natural and synthetic CJ-15,801}

\begin{tabular}{cccc}
\multicolumn{2}{c}{${ }^{1} \mathrm{H}$ NMR $(\mathrm{ppm})$} & \multicolumn{2}{c}{${ }^{13} \mathrm{C}$ NMR $(\mathrm{ppm})$} \\
Natural & Synthetic $(400 \mathrm{MHz})$ & Natural & Synthetic $(75.0 \mathrm{MHz})$ \\
$\left(\mathrm{CD}_{3} \mathrm{OD}\right.$ at $\left.3.30 \mathrm{ppm}\right)$ & $\left(\mathrm{CD}_{3} \mathrm{OD}\right.$ at $\left.3.31 \mathrm{ppm}\right)$ & $\left(\mathrm{CD}_{3} \mathrm{OD}\right.$ at $\left.49.8 \mathrm{ppm}\right)$ & $\left(\mathrm{CD}_{3} \mathrm{OD}\right.$ at $\left.49.2 \mathrm{ppm}\right)$ \\
$7.94(1 \mathrm{H}, \mathrm{d}, J=14.3 \mathrm{~Hz})$ & $7.96(1 \mathrm{H}, \mathrm{d}, J=14.4 \mathrm{~Hz})$ & 175.8 & 175.3 \\
$5.69(1 \mathrm{H}, \mathrm{d}, J=14.3 \mathrm{~Hz})$ & $5.70(1 \mathrm{H}, \mathrm{d}, J=14.4 \mathrm{~Hz})$ & 172.4 & 172.0 \\
$4.02(1 \mathrm{H}, \mathrm{s})$ & $4.02(1 \mathrm{H}, \mathrm{s})$ & 139.8 & 139.3 \\
$3.48(1 \mathrm{H}, \mathrm{d}, J=11.1 \mathrm{~Hz})$ & $3.50(1 \mathrm{H}, \mathrm{d}, J=10.8 \mathrm{~Hz})$ & 104.0 & 103.5 \\
$3.37(1 \mathrm{H}, \mathrm{d}, J=11.1 \mathrm{~Hz})$ & $3.39(1 \mathrm{H}, \mathrm{d}, J=10.8 \mathrm{~Hz})$ & 77.9 & 77.3 \\
$0.93(3 \mathrm{H}, \mathrm{s})$ & $0.93(3 \mathrm{H}, \mathrm{s})$ & 70.6 & 70.0 \\
$0.93(3 \mathrm{H}, \mathrm{s})$ & $0.92(3 \mathrm{H}, \mathrm{s})$ & 41.6 & 40.9
\end{tabular}

$[\alpha]_{D}$ of Natural and synthetic CJ-15,801:

\begin{tabular}{|c|c|c|}
\hline & Natural CJ-15,801 & Synthetic CJ-15,801 \\
\hline$[\alpha]_{\mathrm{D}}$ & $+47.7^{\mathrm{o}}(c 0.44, \mathrm{MeOH})$ & $+54.3^{\circ}(c 0.44, \mathrm{MeOH})$ \\
\hline
\end{tabular}


${ }^{1} \mathrm{H}$ NMR (300 MHz, CD $\mathrm{CD}_{3} \mathrm{OD}$ ) of natural CJ-15,801

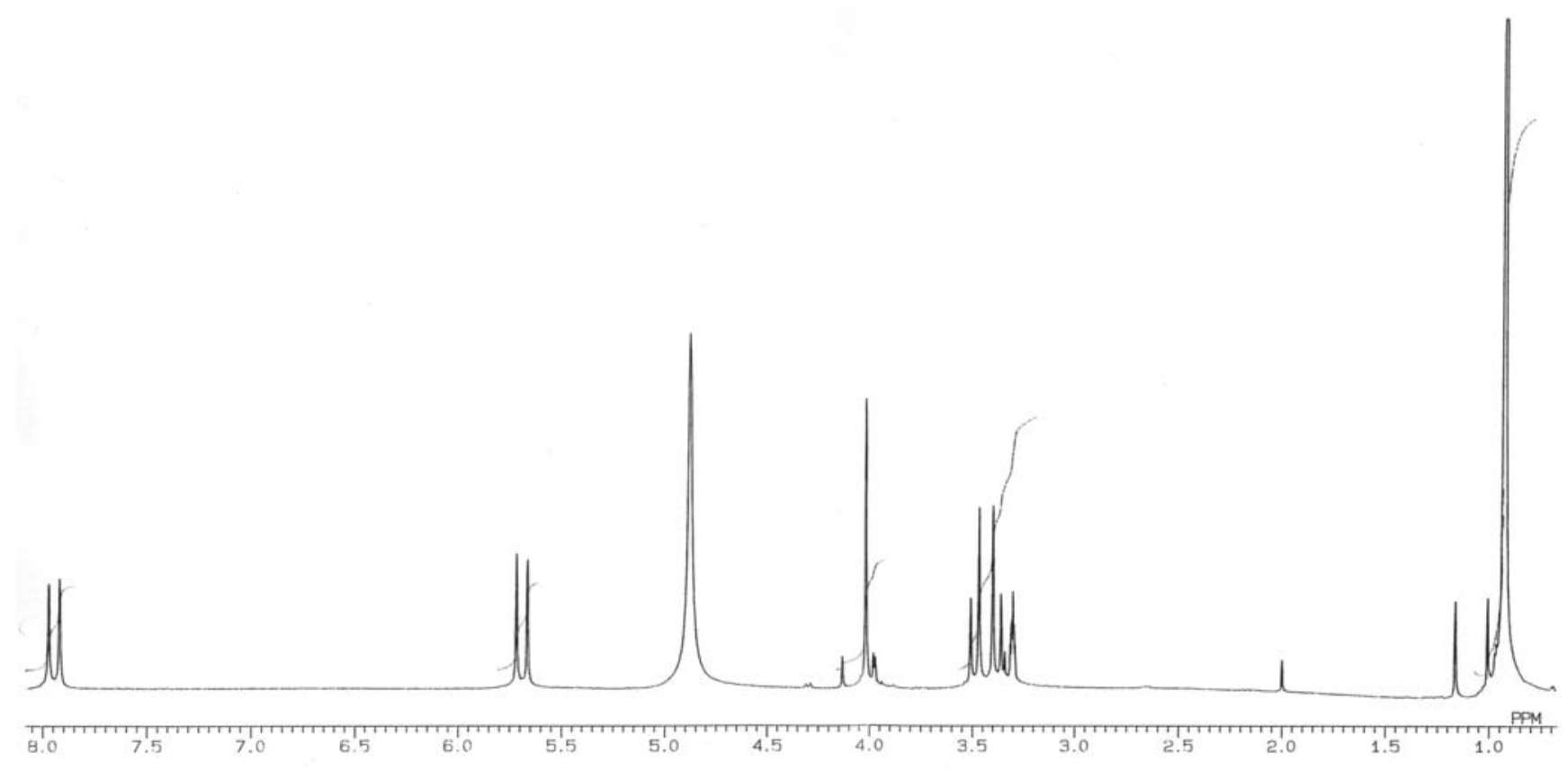

${ }^{1} \mathrm{H}$ NMR (400 MHz, CD ${ }_{3} \mathrm{OD}$ ) of synthetic CJ-15,801

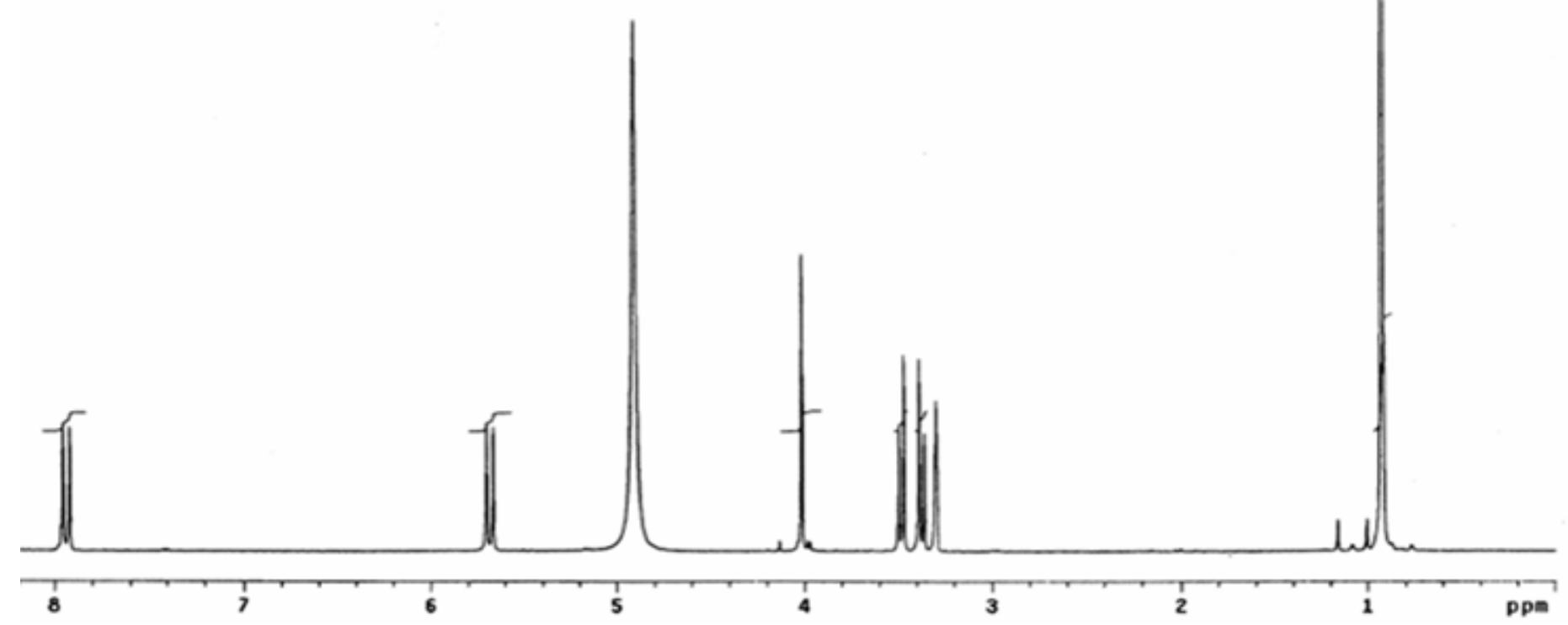


${ }^{13} \mathrm{C}$ NMR (67.5 MHz, $\left.\mathrm{CD}_{3} \mathrm{OD}\right)$ of natural CJ-15,801

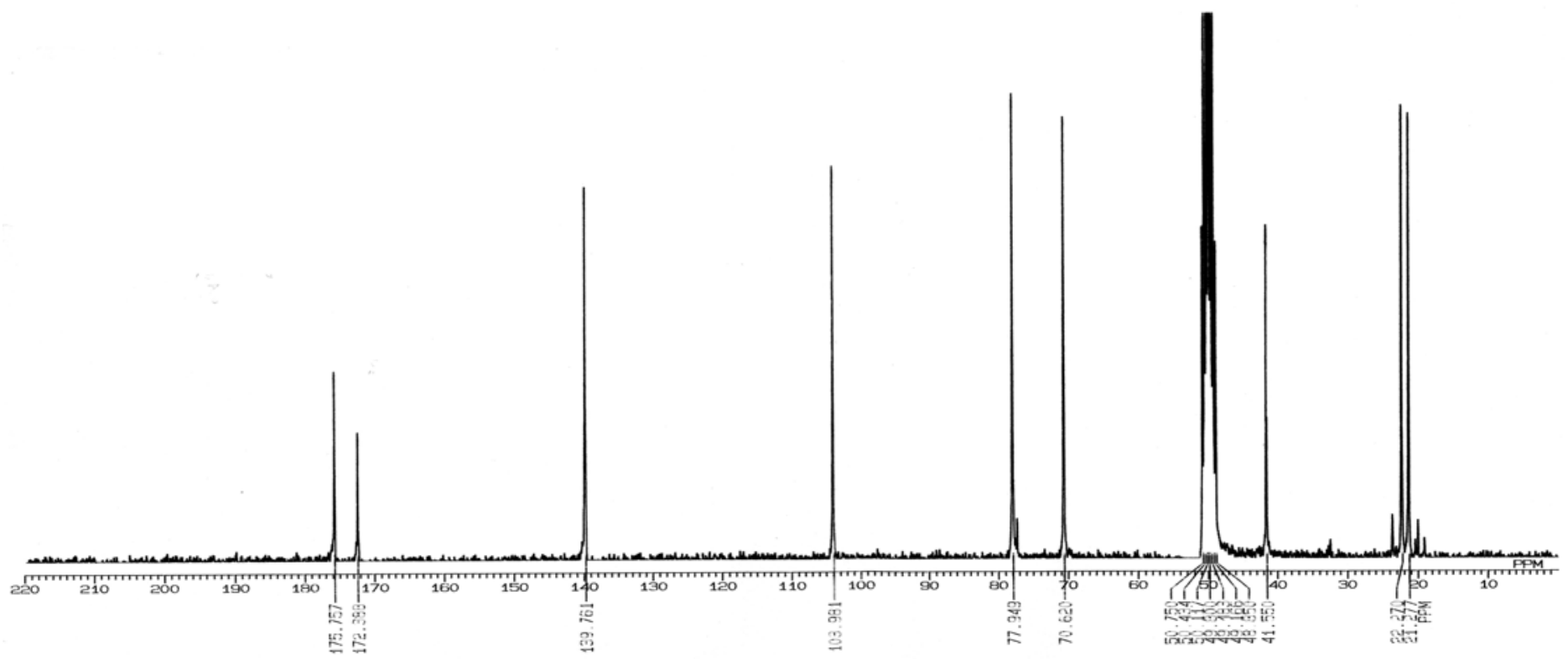

${ }^{13} \mathrm{C}$ NMR (75 MHz, CD ${ }_{3} \mathrm{OD}$ ) of synthetic CJ-15,801

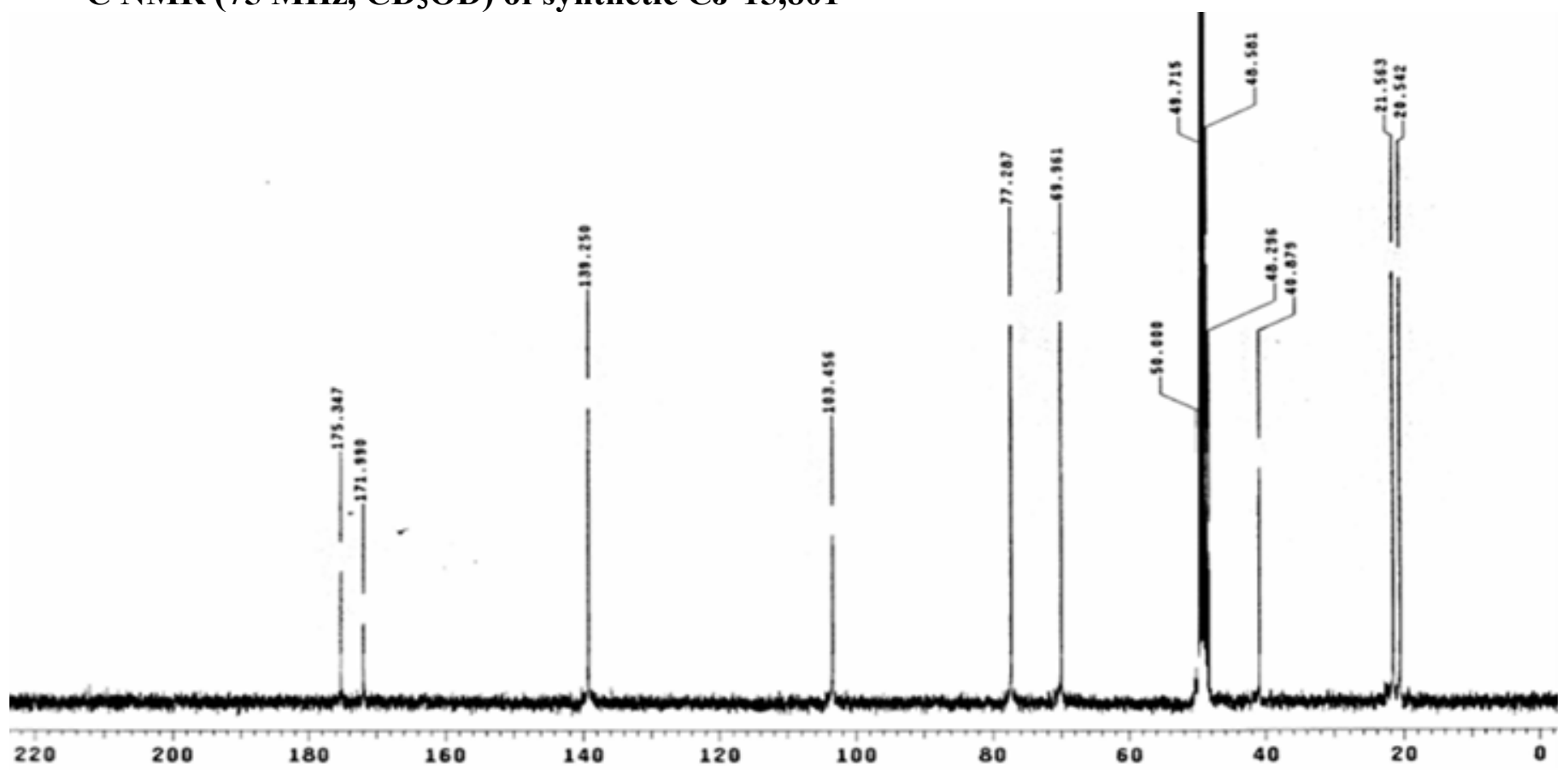




\section{HPLC data for natural and synthetic CJ-15,801 ${ }^{a}$}

\section{Natural:}

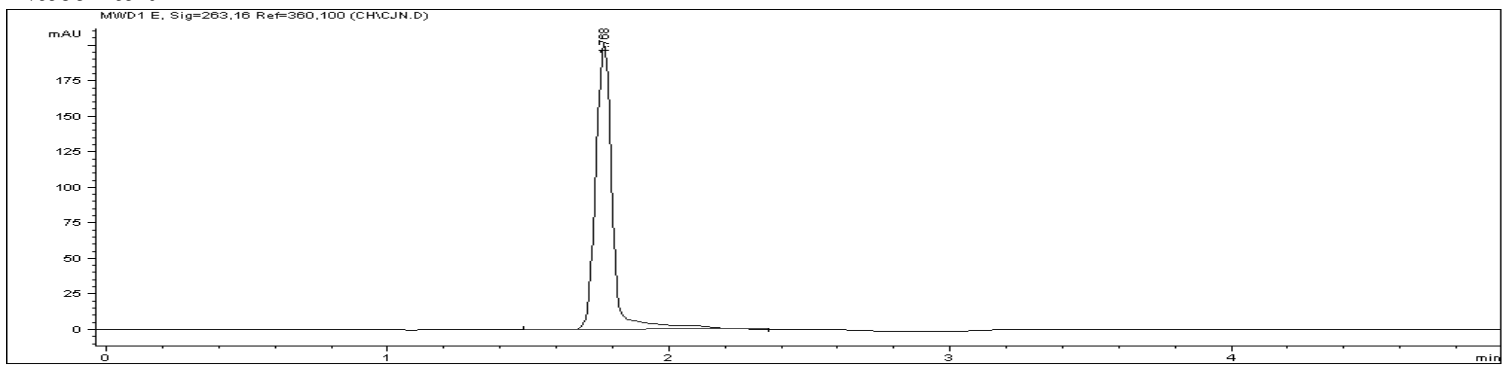

\section{Synthetic:}

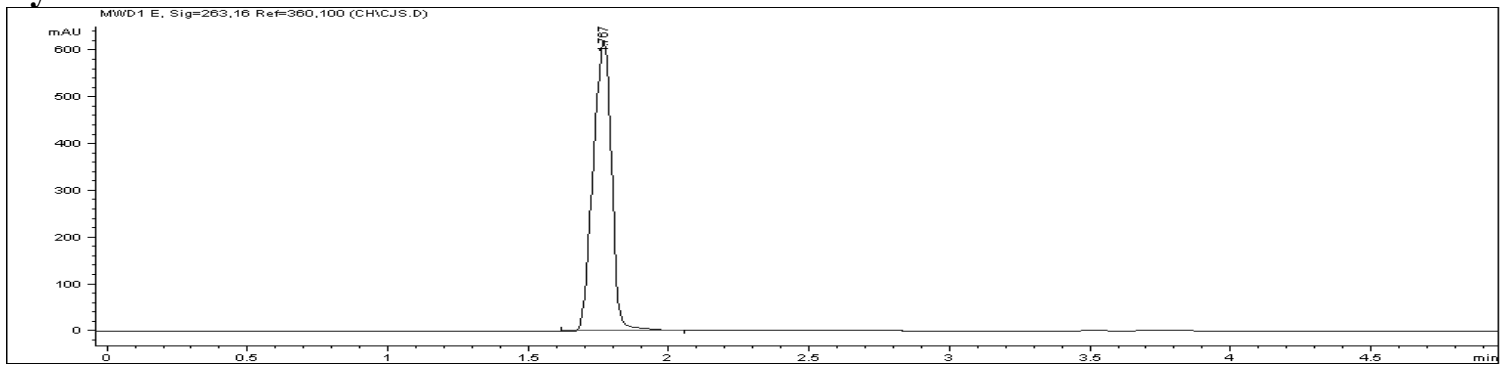

\section{Natural + Synthetic:}

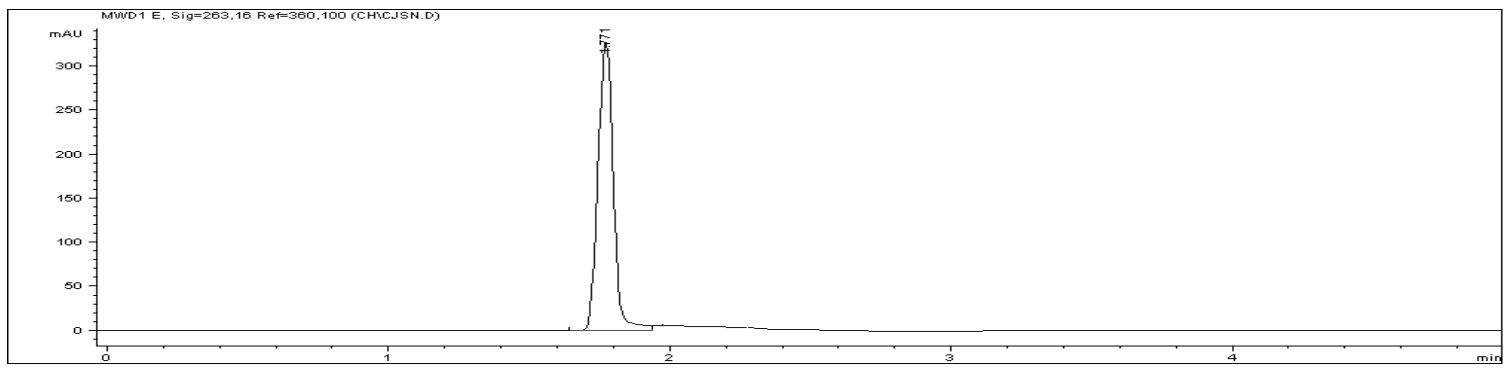

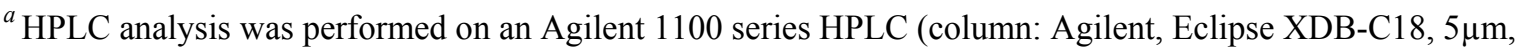
$4.6 \times 150 \mathrm{~mm}$ ). Conditions: $5 \% \mathrm{CH}_{3} \mathrm{CN}$ in $\mathrm{H}_{2} \mathrm{O}$, flow rate: $1 \mathrm{~mL} / \mathrm{min}$ 


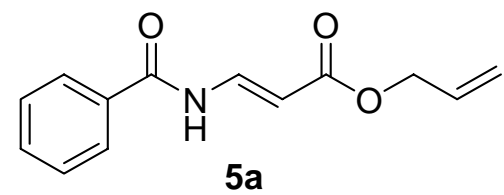

${ }^{1}$ H NMR (400 MHz, $\mathrm{CDCl}_{3}$ )

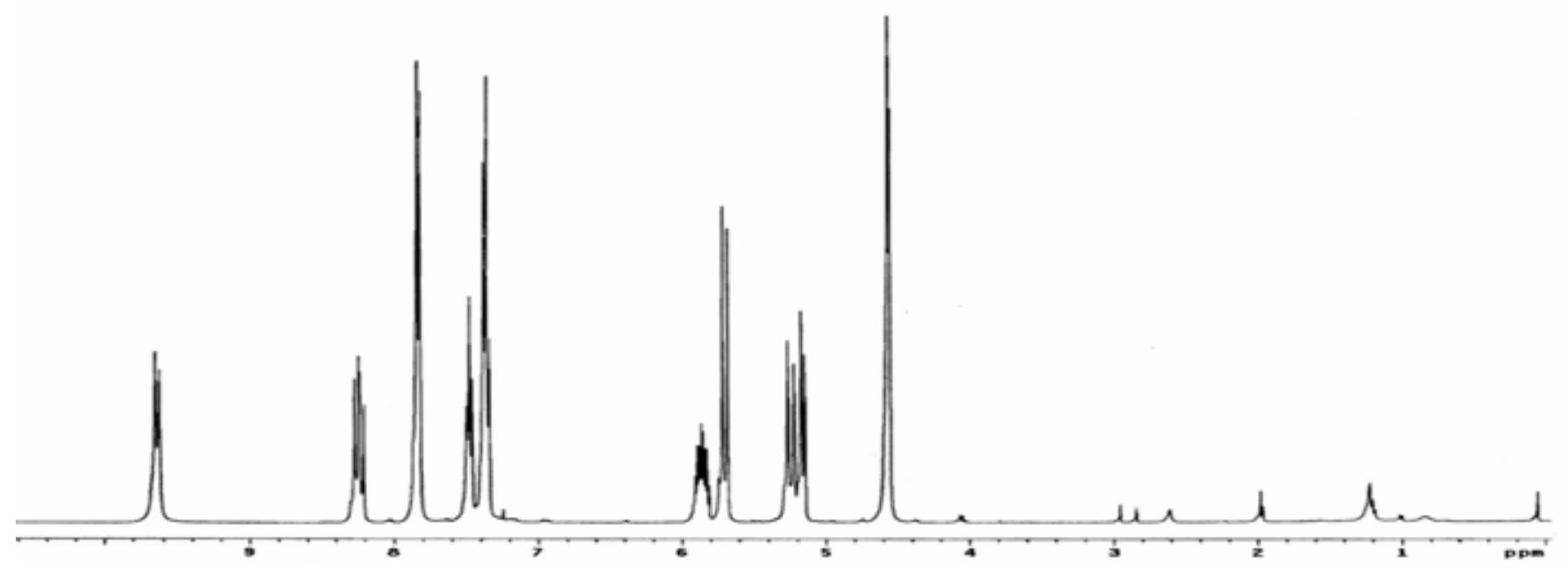

${ }^{13} \mathrm{C}$ NMR (75 MHz, CDCl 3 )

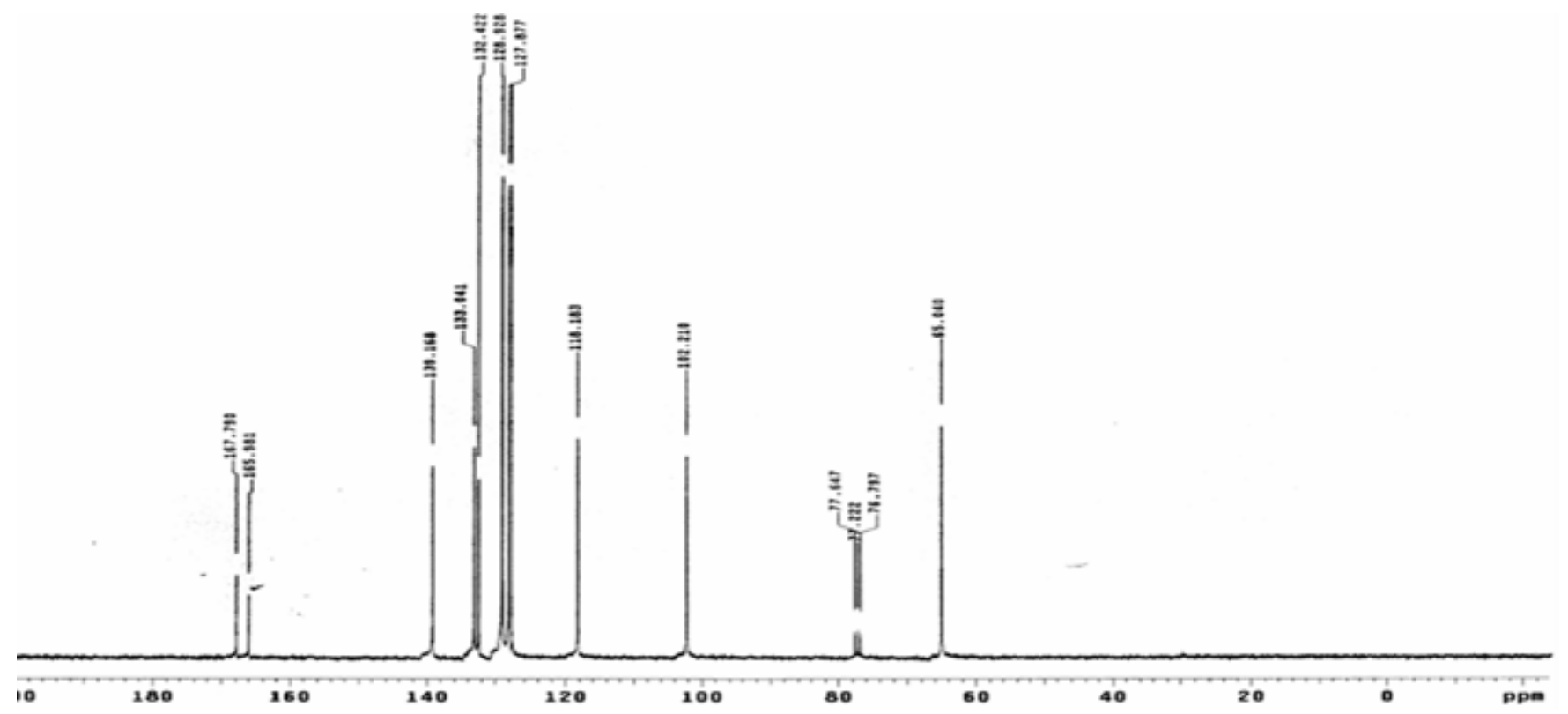




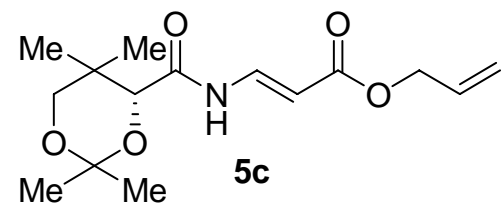

${ }^{1} \mathrm{H}$ NMR (400 MHz, $\mathrm{CDCl}_{3}$ )

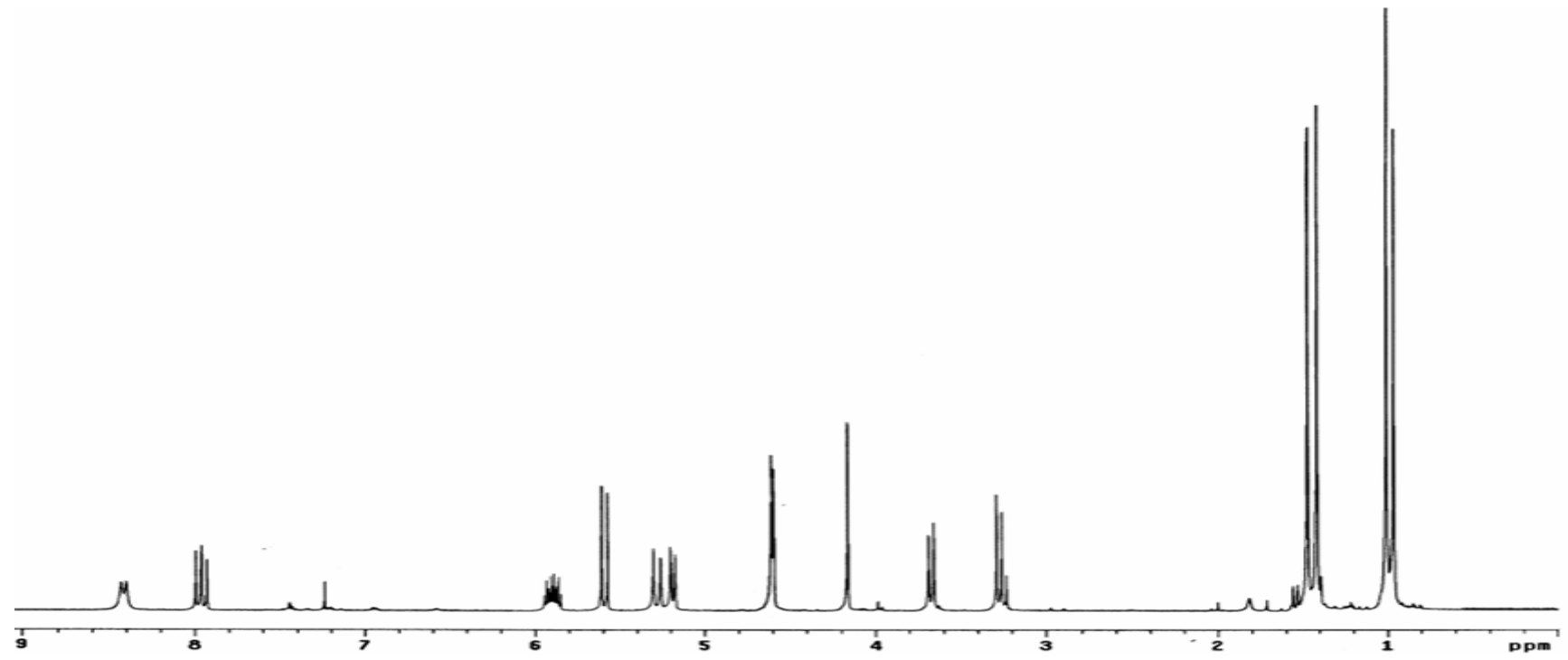

${ }^{13} \mathrm{C}$ NMR (75 MHz, $\mathrm{CDCl}_{3}$ )

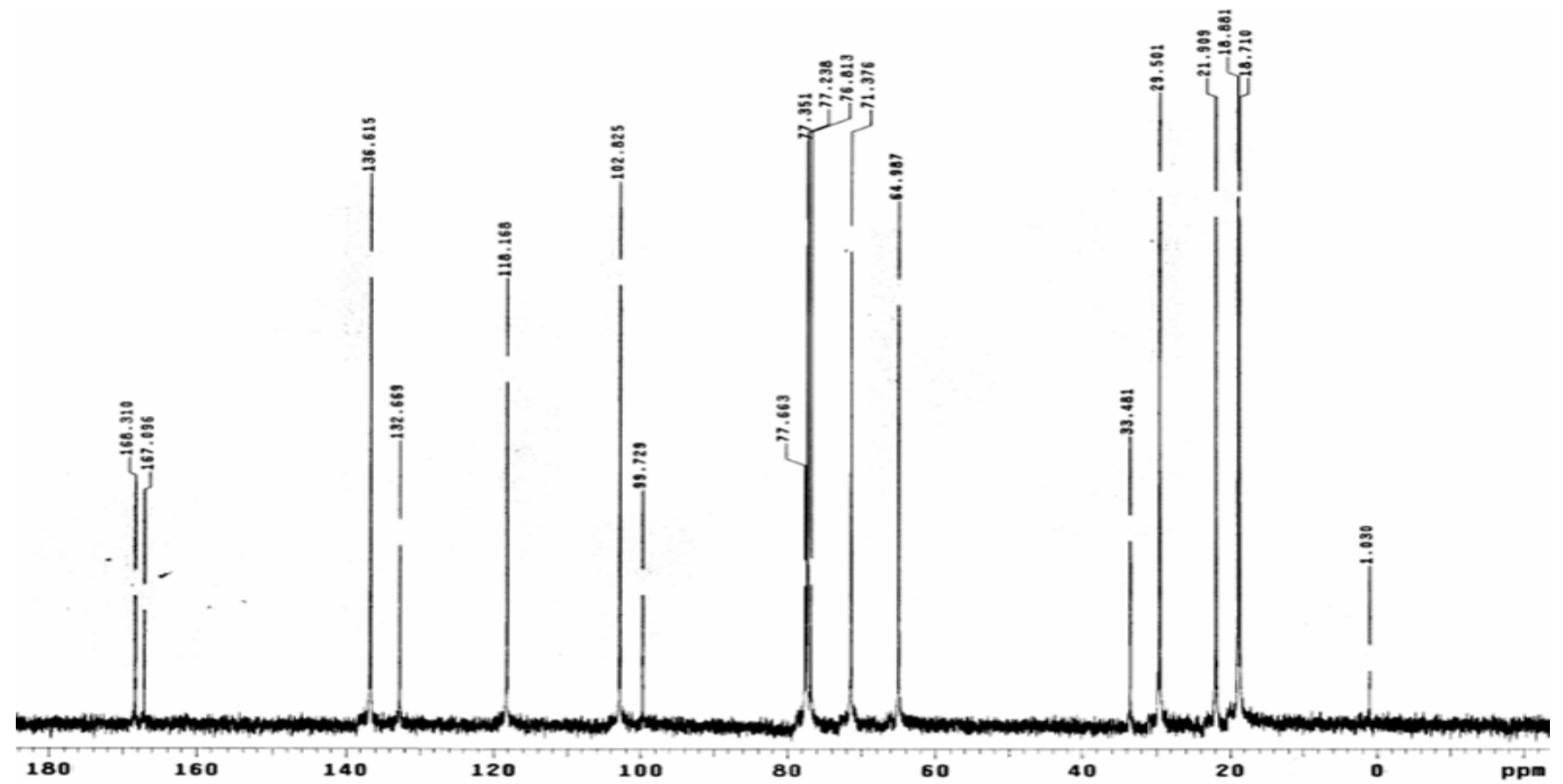




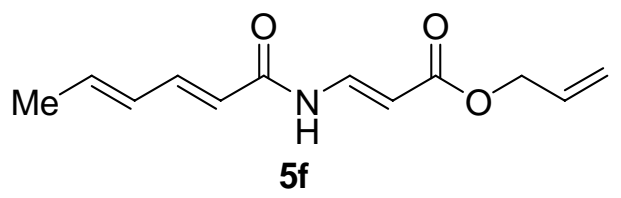

${ }^{1}$ H NMR (400 MHz, $\mathrm{CDCl}_{3}$ )

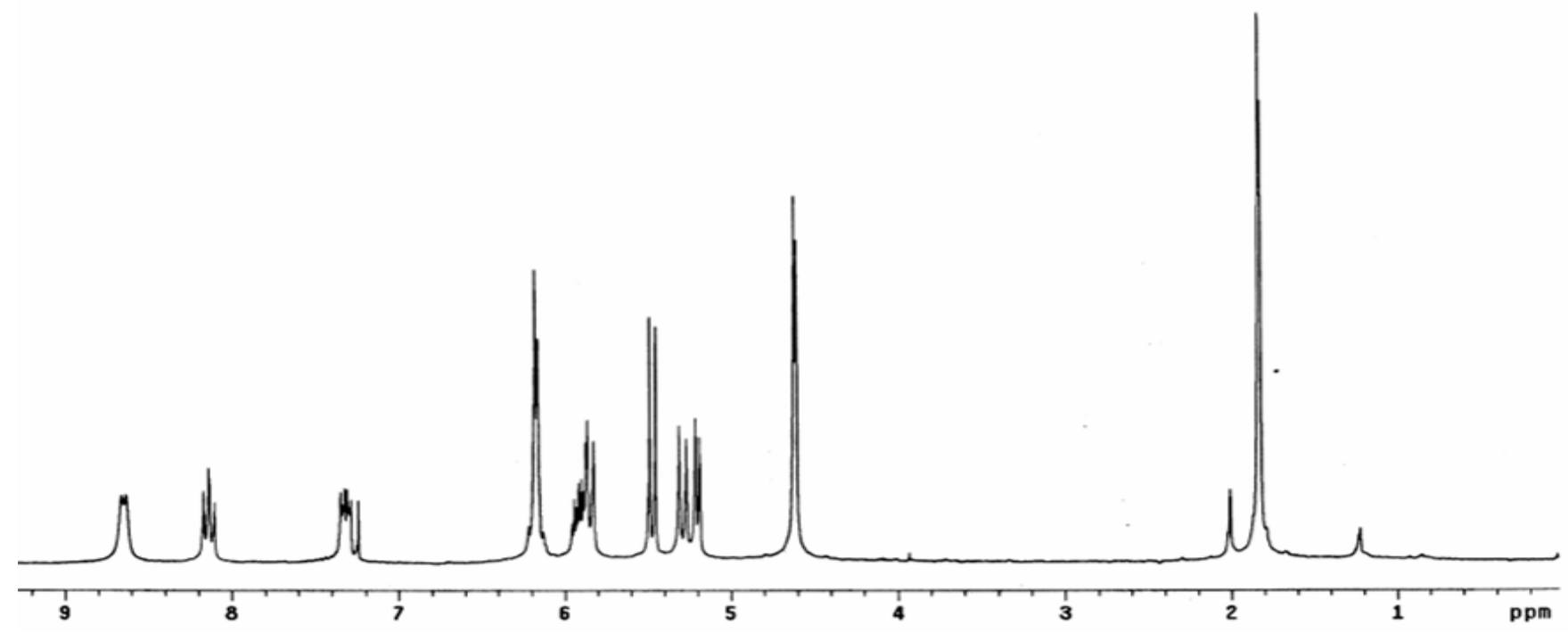

${ }^{13} \mathrm{C}$ NMR (75 MHz, $\mathrm{CDCl}_{3}$ )

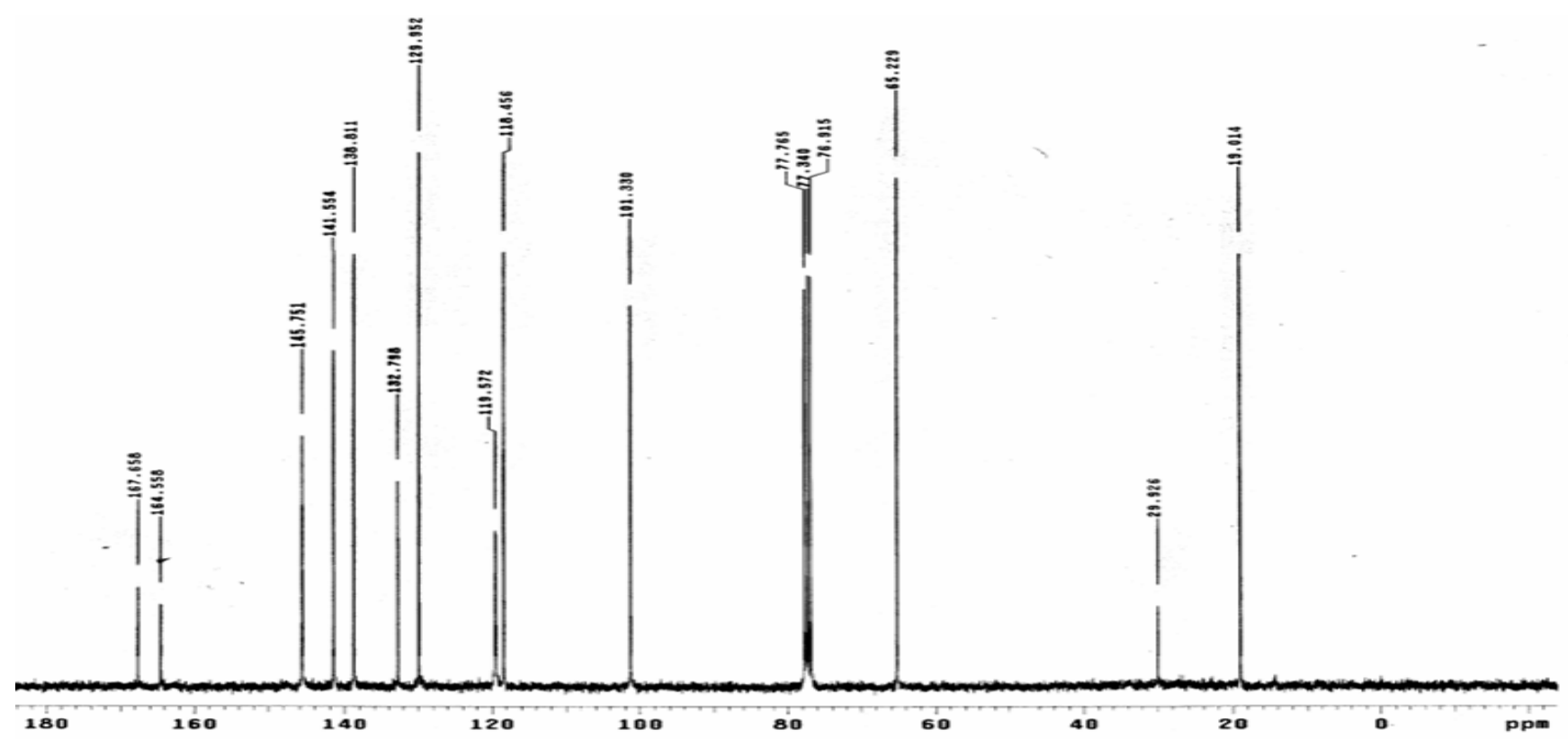


<smiles>O=C(/C=C/NC(=O)c1ccccc1)NCc1ccccc1</smiles>

${ }^{1}$ H NMR (400 MHz, CD 3 OD)

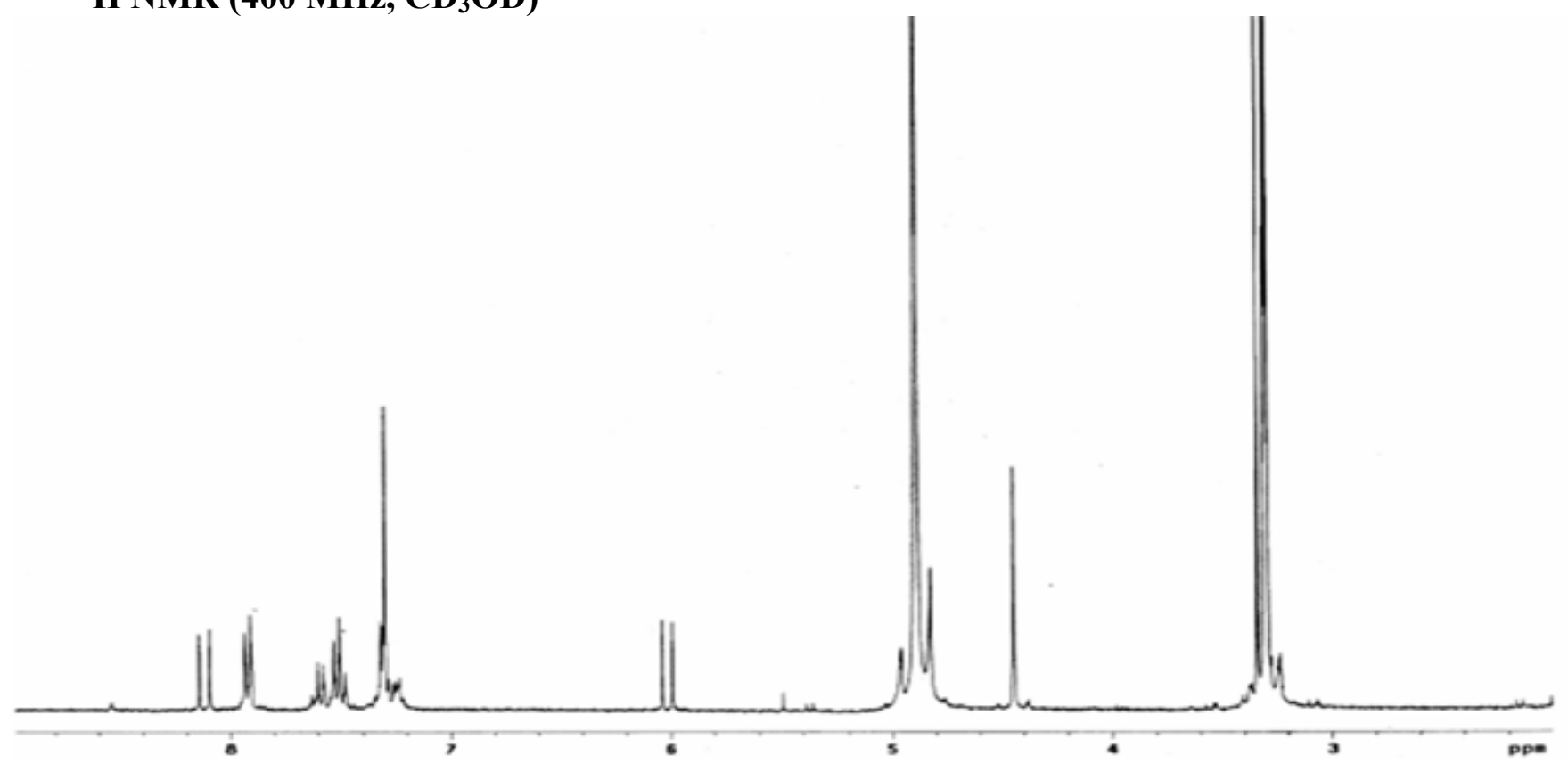

${ }^{13}$ C NMR (75 MHz, DMSO-d $\left.{ }_{6}\right)$

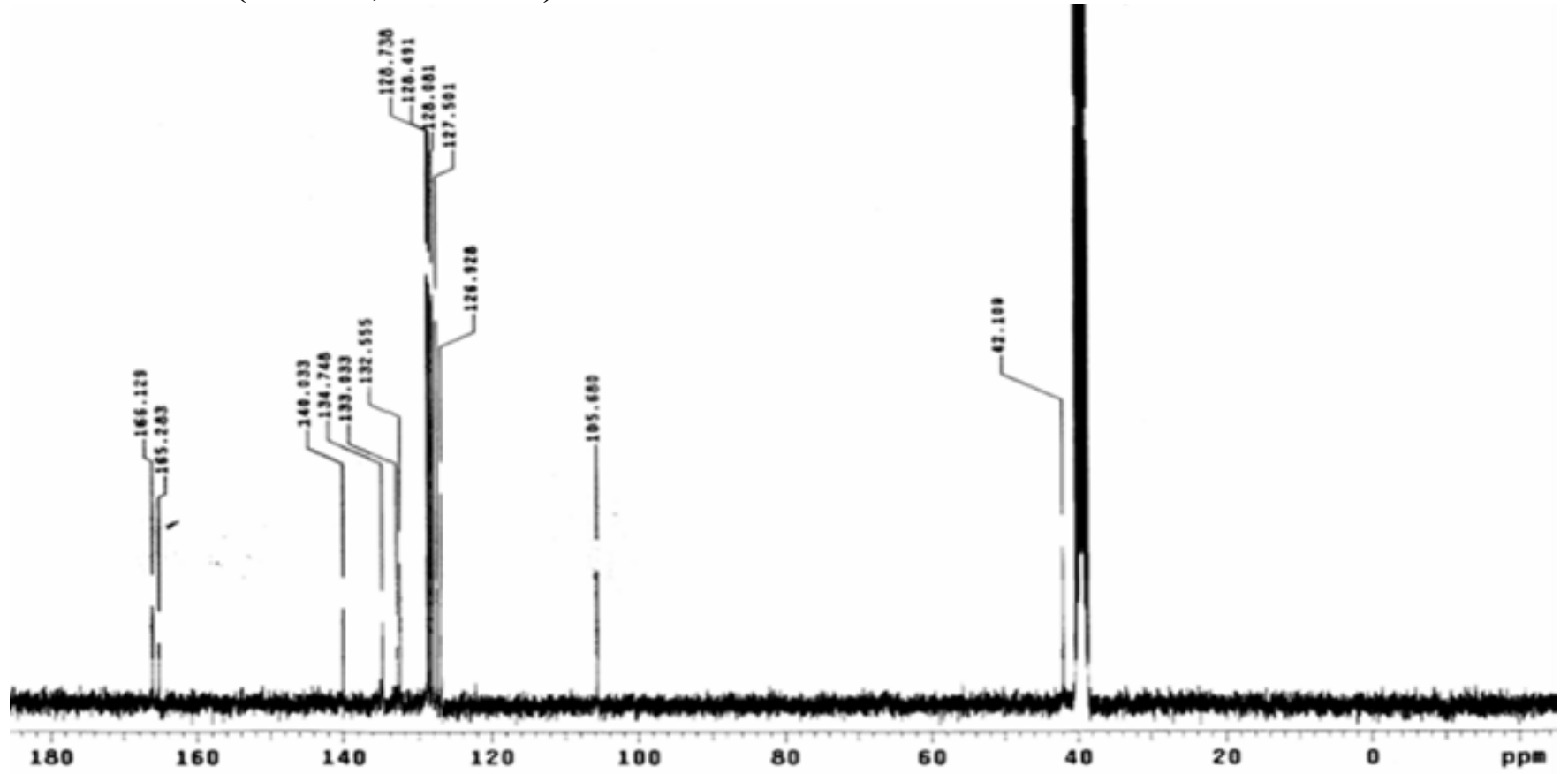


<smiles>C/C=C/C=C/C(=O)N/C=C/C(=O)NCc1ccccc1</smiles>

$11 b$

${ }^{1}$ H NMR (400 MHz, acetone- $d_{6}$ )

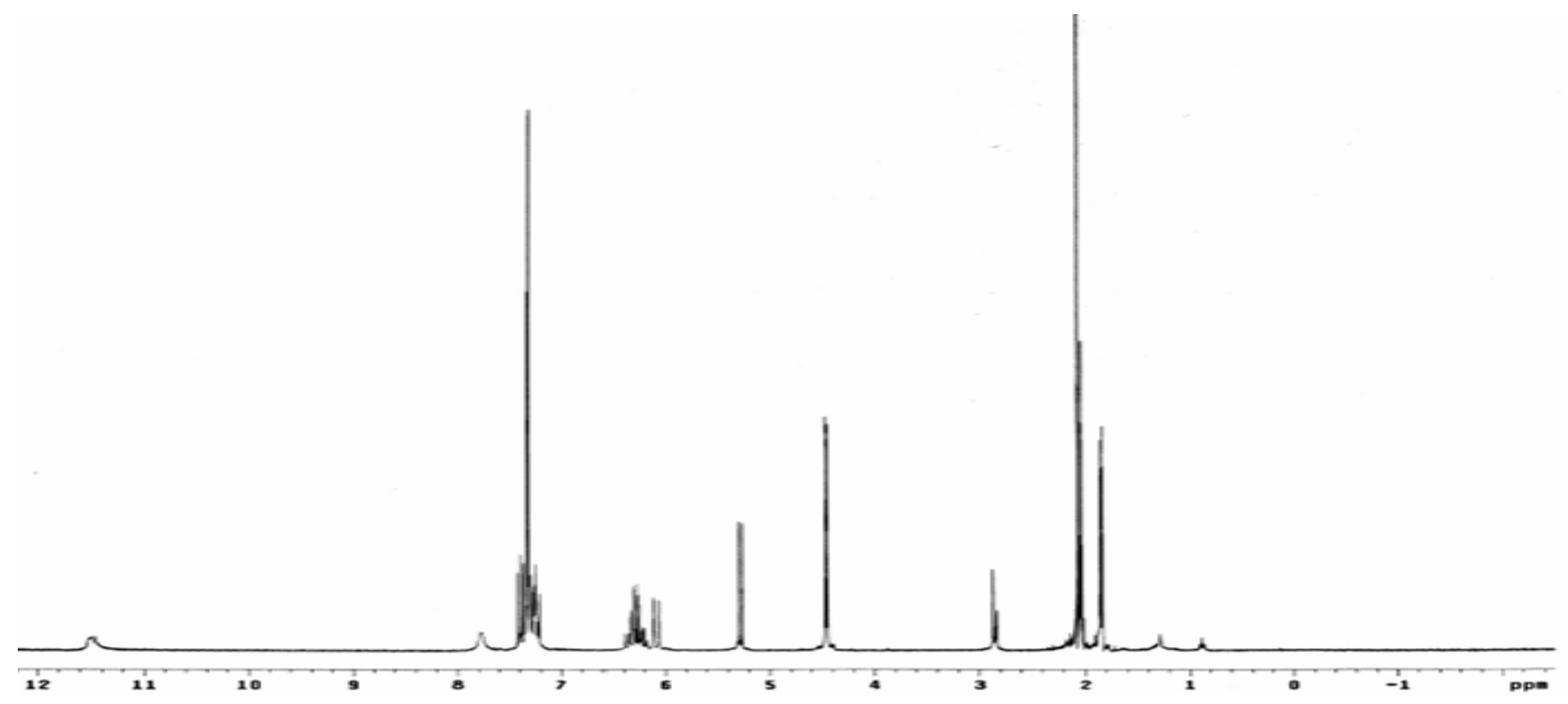

${ }^{13}$ C NMR (75 MHz, acetone- $\left.d_{6}\right)$

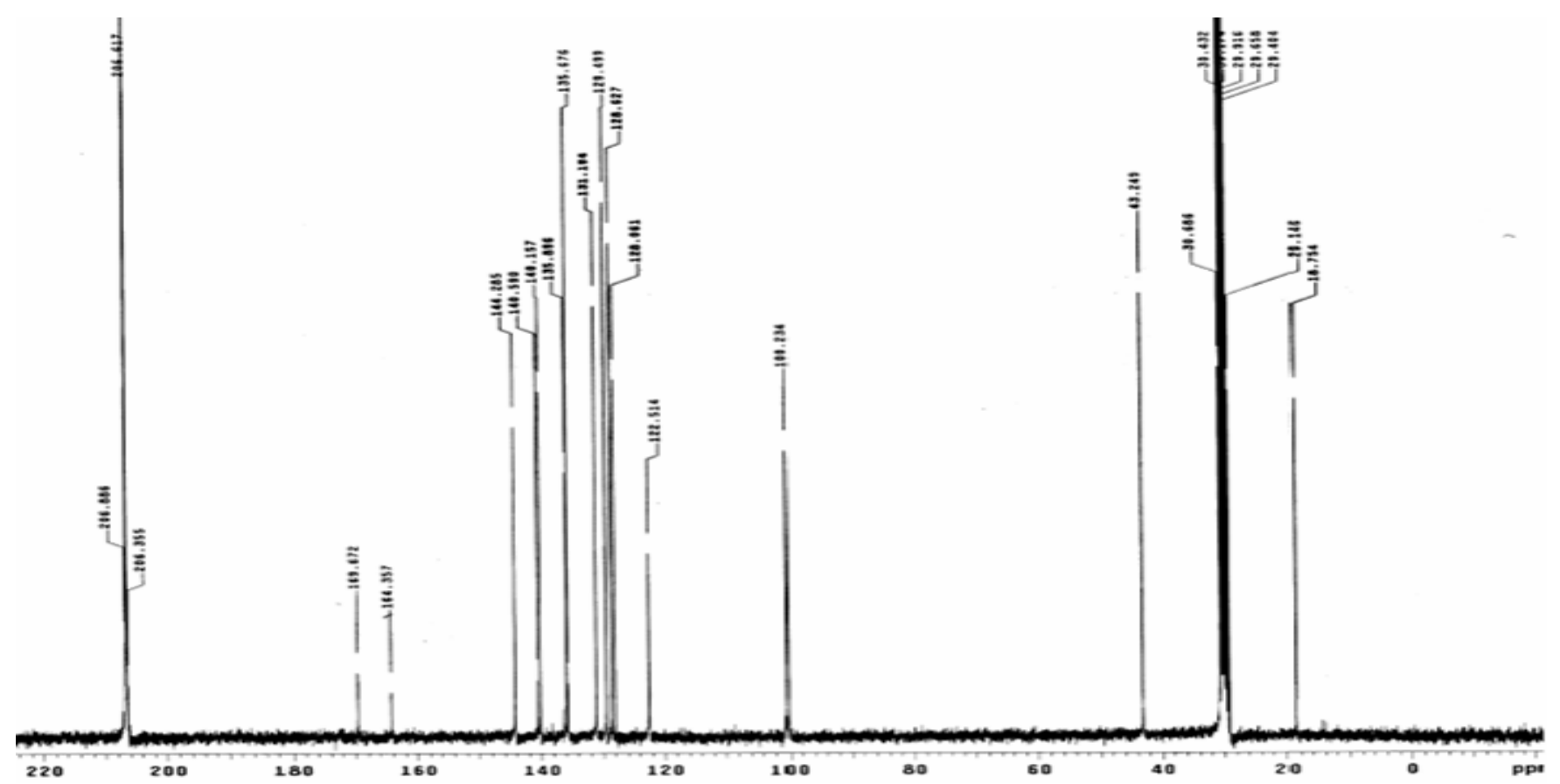


<smiles>C=CCOC(=O)/C=C/NC(=O)[C@@H](O)C(C)(C)CO</smiles>

${ }^{1}$ H NMR (400 MHz, CD ${ }_{3}$ OD)

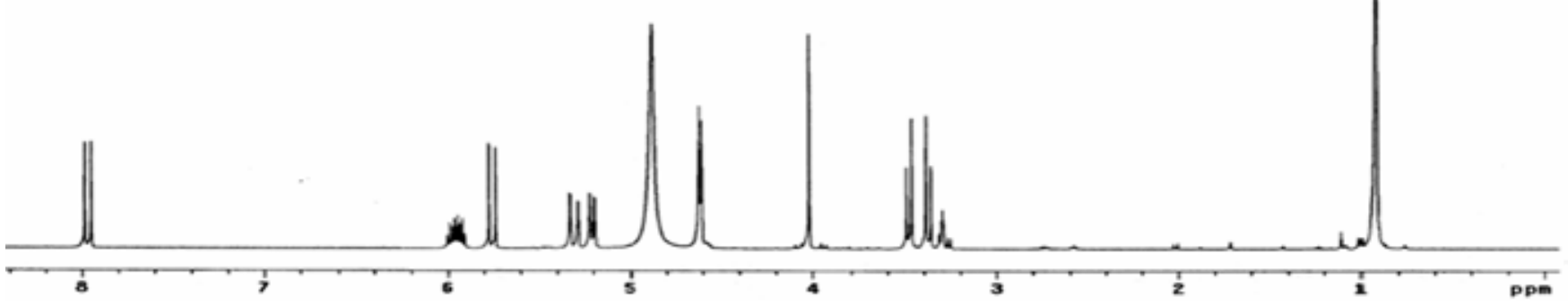

${ }^{13} \mathrm{C}$ NMR (75 MHz, CD 3 OD)

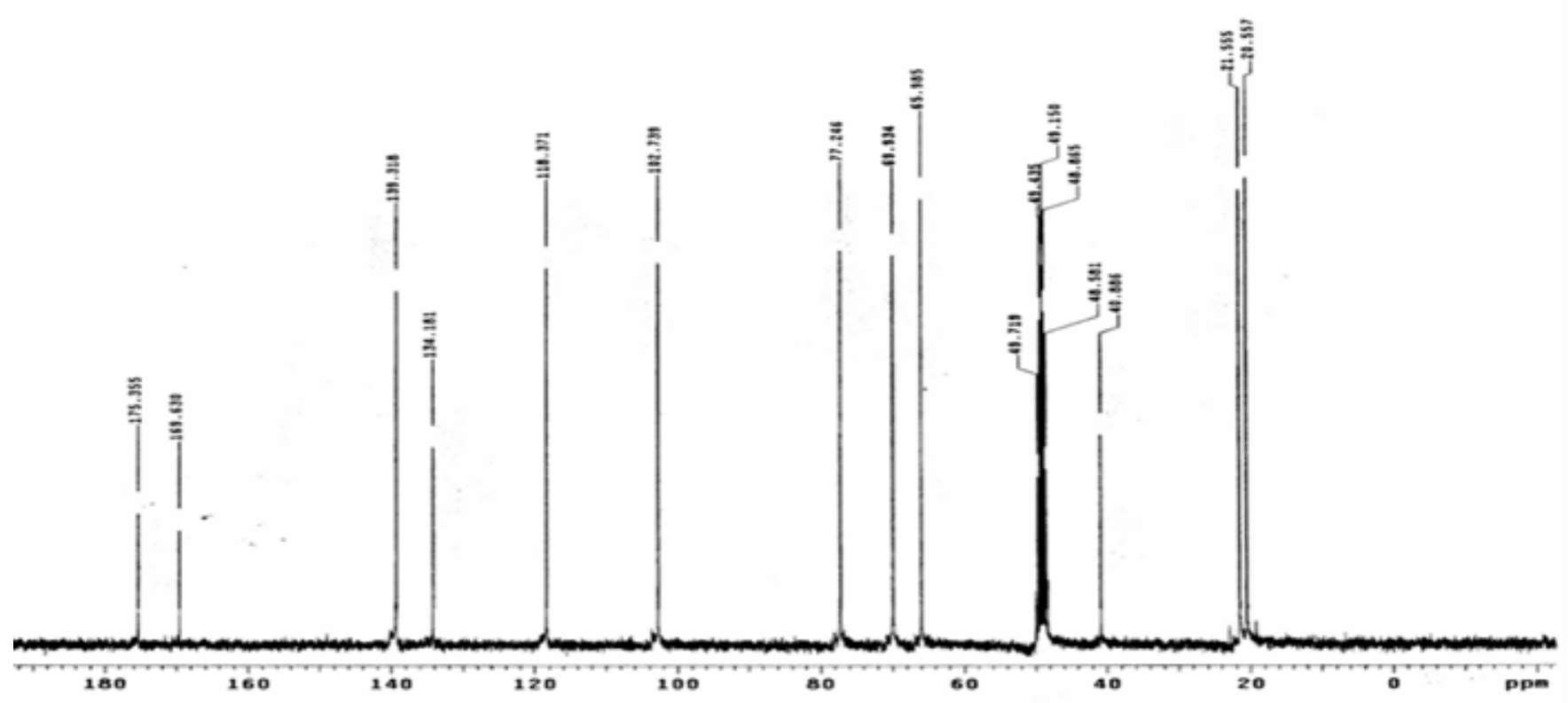

S19 


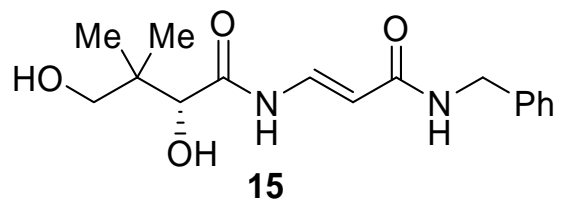

${ }^{1}$ H NMR (400 MHz, acetone- $d_{6}$ )

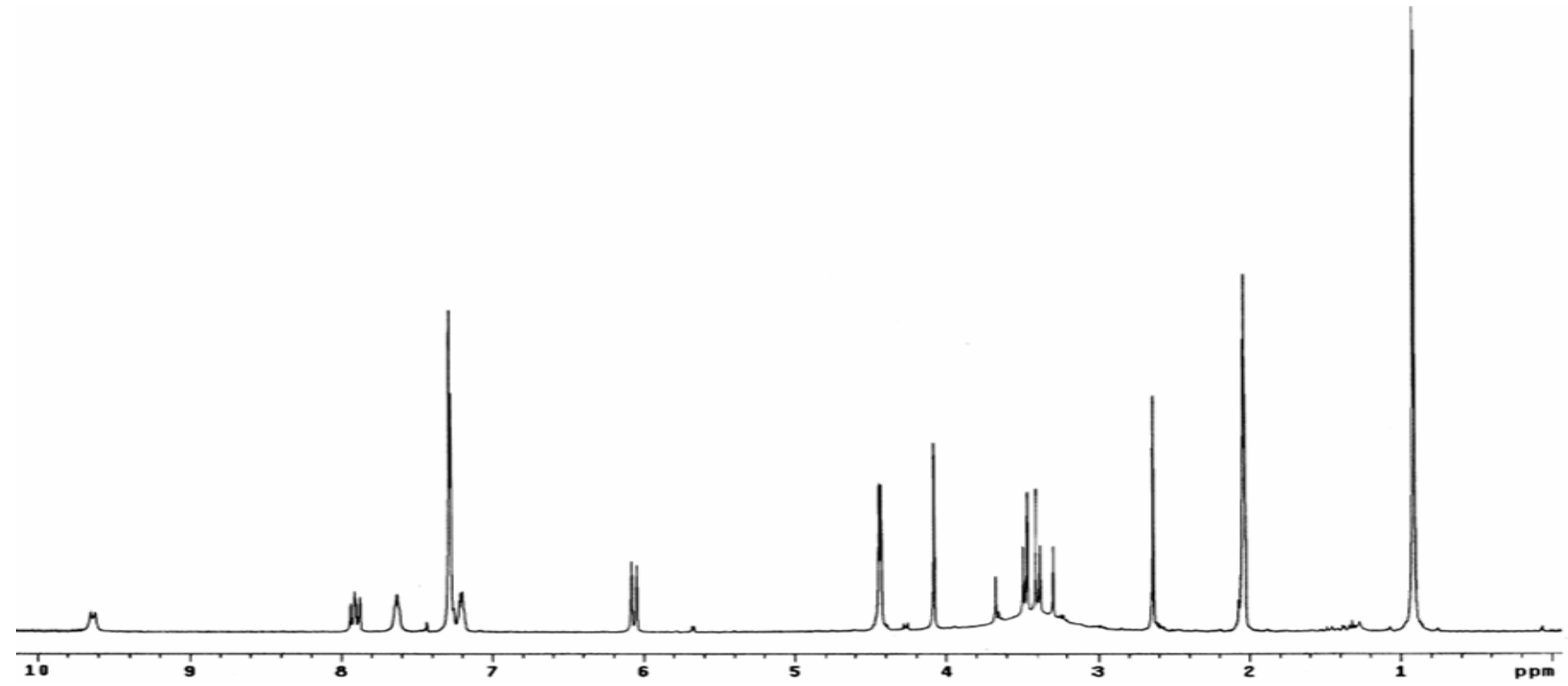

${ }^{13} \mathrm{C}$ NMR (75 MHz, acetone- $d_{6}$ )

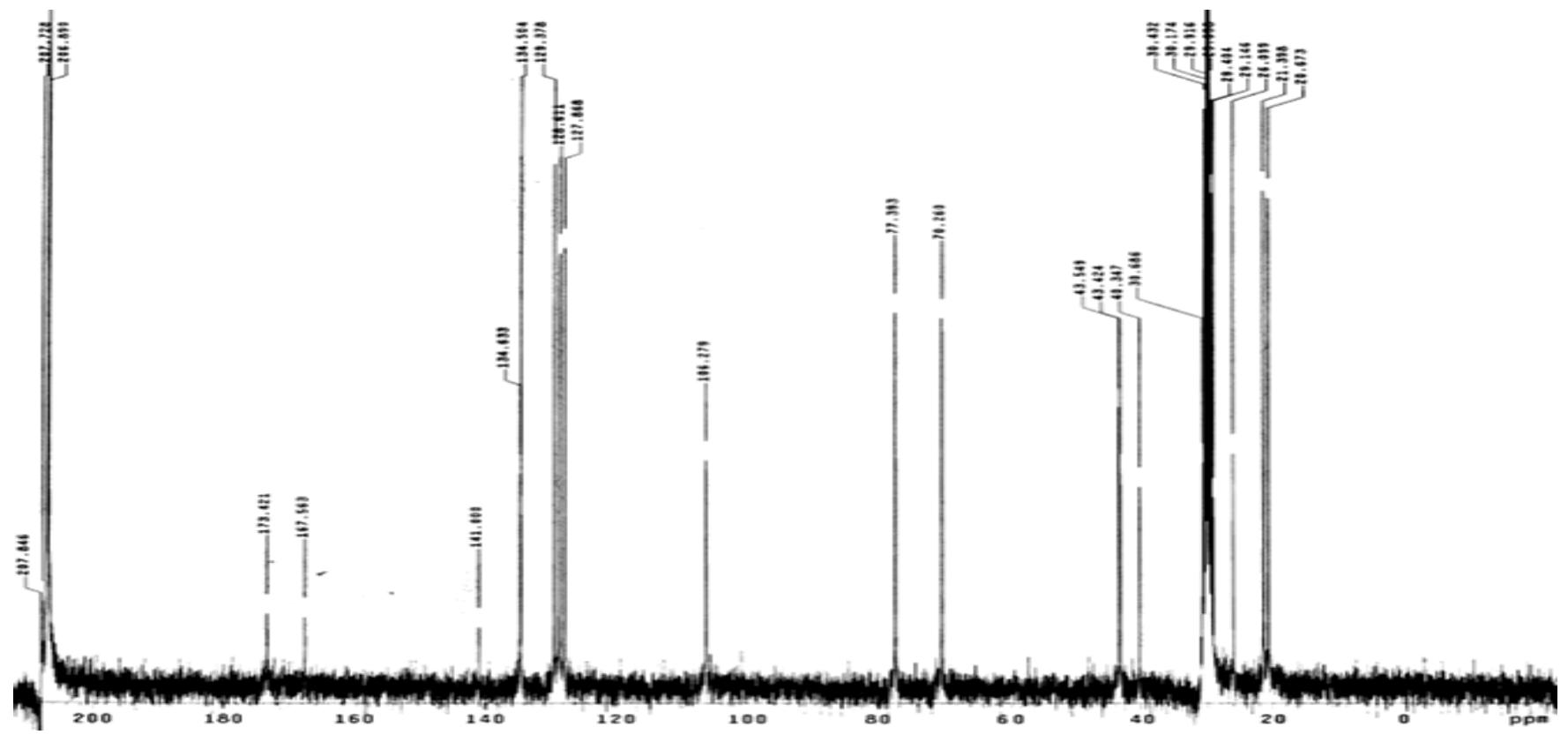

